\title{
Multicast Routing Protocols in MANET
}

\author{
Javad Akbari Torkestani \\ Department of Computer Engineering, Islamic Azad University, Arak Branch, Arak, Iran \\ j-akbari@iau-arak.ac.ir
}

Mohammad Reza Meybodi

Department of Computer Engineering and IT, Amirkabir University of Technology, Tehran, Iran

mmeybodi@aut.ac.ir

\begin{abstract}
Multicast routing is an effective way to establish the group communication when the same message or the same stream of data needs to be sent to multiple receivers. Multicast routing has attracted a lot of attention in group oriented computing due to supporting data transmission from a single source node to multiple destinations concurrently. The advantage of multicast routing lies in its capability of reducing the communication cost and saving the network resources by sending only one copy of the message over the shared link leading to different destinations. Generally speaking, this survey classifies the multicast routing protocols into four categories based on the underlying routing structure: tree-based, mesh-based, hybrid and stateless multicast routing protocols. This survey summarizes the well-known most recent protocols of each category and compares their objectives, performances, advantages and disadvantages. This survey study helps us to choose the best multicasting protocol for each network application with respect to the requirements of application.
\end{abstract}

Keyword Multicast routing, mobile ad hoc network, tree-based protocol, meshbased protocol, on-demand protocol, proactive protocol 


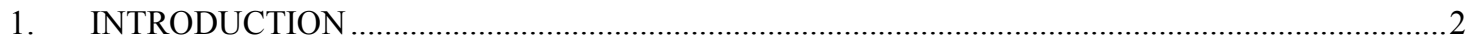

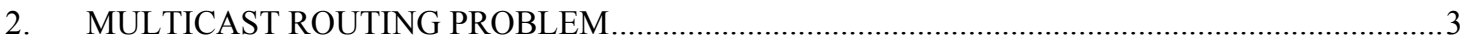

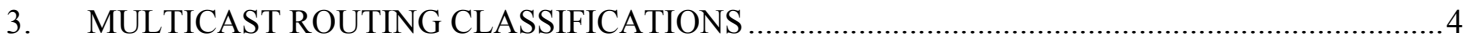

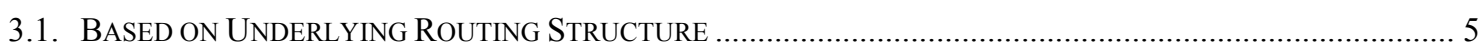

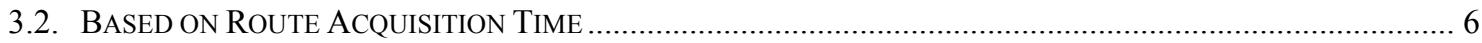

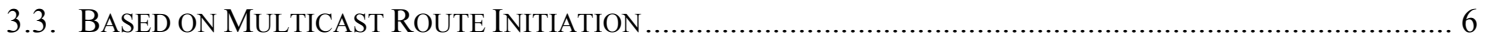

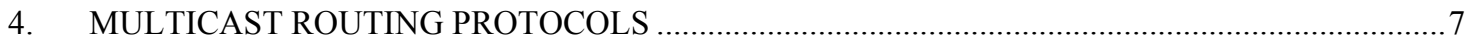

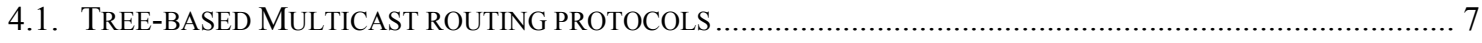

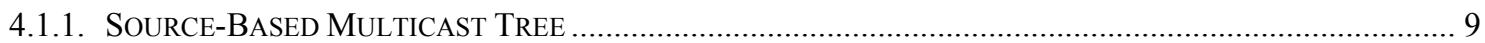

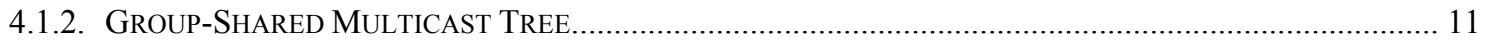

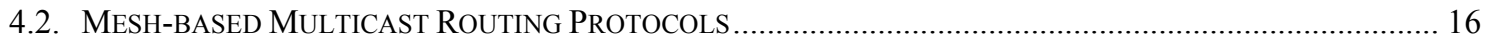

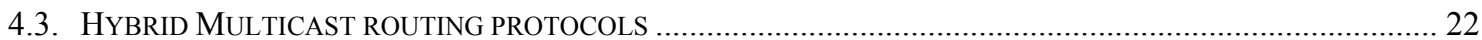

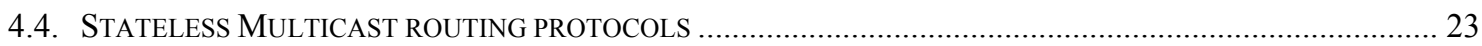

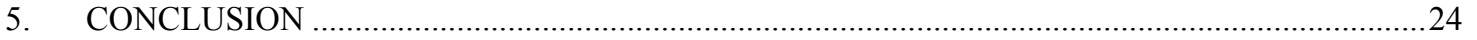

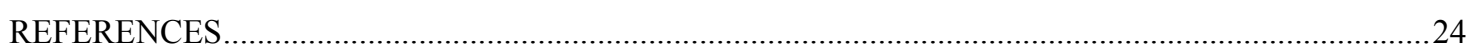

\section{Introduction}

Multicasting is a technique for data routing in networks that allows the same message is forwarded to a group of destinations simultaneously. In mobile ad hoc networks (MANETs), the most challenging issue in multicast routing is to effectively handle the frequent and unpredictable topology changes caused by host mobility, link breakage and host failure. Multicasting is intended for grouporiented computing like audio/video conferencing, collaborative works, and etc. Multicasting is an essential technology to efficiently support one-to-many or many-to-many applications. Multicast routing has attracted a lot of attention in the past decade, due to it allows a source to send information to multiple destinations concurrently. Multicasting is the transmission of packets to a group of zero or more hosts called multicast group which is identified by a single destination address. A multicast group is a set of network clients and servers interested in sharing a specific set of data. A typical example of multicast groups is a commander and his soldiers in a battlefield. There are other examples in which multicast groups need to be established. Typically, the membership of a host group is dynamic: that is, the hosts may join and leave groups at any time. There is no restriction on the location or number of members in a host group. A host may be a member of more than one group at a time. A host does not have to be a member of a group to send packets to it. A multicast protocol has the objective of connecting members of the multicast group in an optimal way, by reducing the amount of bandwidth necessary but also considering other issues such as communication delays and reliability [1].

Multicast routing plays a critical role in most of the new applications such as web-base learning, video conference, and interactive multimedia games. Multicast routing in mobile ad hoc networks poses several challenges due to inherent characteristics of the network such as node mobility, reliability, and scarce resources. The main difficulty in designing a routing protocol for mobile ad hoc networks is the dynamically changing topology, due to the random movement of mobile nodes. The multicast routing protocols designed for wireless mobile ad hoc networks are fundamentally different from those for conventional infrastructure based networks in that these are self-configuring and 
formed directly by a set of mobile nodes without relying on any established infrastructure. In such networks, the heterogeneity of the hosts makes it difficult to achieve bandwidth efficiency and service flexibility.

In ad hoc networks, there exist several methods upon which classification of the multicast routing protocols is based. Classification based on the underlying structure of the multicast routes, based on the route acquisition time, based on multicast route initiation (based on the responsibility for route construction), and based on the forwarding state maintenance schemes are the well-known representative methods. Underlying multicast route structure is the most popular approach based on which the multicast routing protocols are classified. This classification considers the connectivity of the forwarding paths through which the multicast receivers are connected. This survey presents a new comprehensive classification of the multicast routing protocols based on the underlying route structure. In this classification, the multicasting protocols are categorized as tree-based multicast routing protocols, mesh-based multicasting protocols, hybrid protocols, and stateless protocols. This survey studies the most recent effective protocols of each category with the emphasis on the objectives, performances, costs, advantages, and drawbacks.

The rest of this paper is organized as follows. The multicast routing problem is defined in Section 2. In Section 3, the multicast routing protocols proposed for ad hoc networks are classified from several points of view. Section 4 presents a new classification of the multicast routing protocols based on the underlying routing structure and compares the existing multicasting schemes from this point of view. Section 5 concludes the paper.

\section{Multicast Routing Problem}

Multicasting is a technique for data routing in networks that allows the same message is forwarded to a group of destinations simultaneously. Multicast routing problem (MRP) is one of the most difficult problems in communication networks in which the source node (or a set of source nodes) is connected to the set of destinations, while some cost function is simultaneously minimized. In a wireless network, due to the broadcast nature of the omnidirectional antennae, a single transmission can be received by all neighbors of the transmitting node [1]. Therefore, the multicast routing protocols designed for the traditional wired networks are not applicable to the wireless networks. In a wired network, the multicast packets are forwarded along the tree edges, and so the multicast routing problem can be defined as a Steiner tree problem where the multicast group members are the terminals (leaf nodes) in the Steiner tree [2]. The problem of finding a Steiner tree is known to be NP-complete [3], even if links have unit cost [4]. It should be noted that in some multicast routing protocols $[5,6]$ the minimum spanning tree problem, which is a well known approach for broadcasting, is also used to model the multicast routing problem. However, in wireless ad hoc networks, owing to the broadcast nature of the wireless channels, the topology graph of such networks can be modeled as an undirected unit disk graph $G\langle V, E\rangle$, where the nodes represent the individual hosts and an edge connects two nodes if the corresponding hosts are within transmission range of each other. In these networks, the Steiner connected dominating set problem [7, 8] is a promising approach for solving the multicast routing problem, where the only multicast group members need to be dominated. The following represents the graph-based mathematical formulations of the minimum multicast routing problem in wireless networks.

Definition 1 Given a unit disk graph $G\langle V, E\rangle$, a source node $s \in V$ called multicast source, and a set of destinations $D \subset V$ called multicast members, the multicast routing problem can be defined as finding the smallest set of relay (intermediate) nodes $F \subset V$ such that set $\{s\} \cup F \cup D$ is connected.

In most of the multicast routing protocols reported in the literature, it is assumed that all the hosts have the same costs, and so the proposed protocols as described in Definition 1 try to minimize the number of relay nodes for optimizing the multicast routes. In these methods, the multicast routing 
problem is defined as finding the minimum size Steiner connected dominating set in which a subset of relay nodes is chosen as dominators to construct a route from the multicast source to each of the multicast receivers. The host degree, average distance (the distance between the host and its neighbors), mobility characteristics (average speed or relative speed), residual energy level, and transmission power are some parameters can be applied as costs to a given host in a mobile wireless ad hoc network. In many applications of the wireless ad hoc networks, the above assumption (i.e., all hosts have the same costs) can not hold true, and reducing the number of relay nodes is not sufficient. In such networks, due to the hosts heterogeneity, host mobility, and strict resource limitations, each wireless host may have a different cost in the multicast tree. Therefore, in the following we represent a vertex-weighted definition of the minimum multicast routing problem where each host has an individual cost.

Definition 2 Given a node-weighted graph $G\langle V, E, W\rangle$ corresponding to the network topology graph, where $V$ denotes the set of nodes, $E$ denotes the edge set, and $W$ denotes the set of weights which is associated with $V$ by function $W: V \rightarrow \mathfrak{R}^{+}$, a source node $s \in V$, and a set of destinations $D \subset$ $V$. Let $M=\left\{m_{1}, m_{2}, \ldots\right\}$ denotes the set of all possible multicast routes by which the multicast source is connected to all the multicast members, and

$$
w_{m_{i}}=\sum_{\forall v_{j} \in m_{i}} w_{v_{j}}
$$

denotes the weight of multicast route $m_{i}$. Multicast route $m^{*} \in M$ is the optimal solution to the MRP, if and only if we have

$$
w_{m^{*}}=\min _{\forall m_{i} \in M} w_{m_{i}}
$$

The above definition formulates the multicast routing problem as the minimum node-weighted Steiner connected dominating set of the network topology graph. Since the characteristics of the mobile ad hoc networks are stochastic, unpredictable and time-varying $[9,10,11]$, stochastic graphs are more appropriate data structures for modeling the ad hoc network topology graphs. Therefore, in what follows, we represent the definition of the stochastic graph to model the ad hoc networks, then, we define the MRP in such networks. A node-weighted graph is a graph in which a value (or weight) is associated with each node. A stochastic node-weighted graph is a node-weighted graph in which the weight associated with each node is a random variable.

Definition 3 Given a node-weighted graph $G\langle V, E, W\rangle$ corresponding to the network topology graph, where $V$ denotes the set of nodes, $E$ denotes the edge set, and $W$ denotes the set of weights which is associated with $V$ by probability distribution function $W: V \rightarrow \mathfrak{R}^{+}$, a source node $s \in V$, and a set of destinations $D \subset V$. Let $M=\left\{m_{1}, m_{2}, \ldots\right\}$ denotes the set of all possible multicast routes by which the multicast source is connected to all the multicast members, and

$$
\bar{w}_{m_{i}}=\sum_{\forall v_{j} \in m_{i}} \bar{w}_{v_{j}}
$$

denotes the expected weight of multicast route $m_{i}$. Multicast route $m^{*} \in M$ is the optimal solution to the MRP, if and only if we have

$$
\bar{w}_{m^{*}}=\min _{\forall m_{i} \in M} \bar{w}_{m_{i}}
$$

That is, the optimal (minimum cost) multicast route in a stochastic network is defined as the multicast route with the minimum expected cost. By Definition 3 the multicast routing problem in a stochastic network is formulated as the stochastic Steiner connected dominating set of the network topology graph.

\section{Multicast Routing Classifications}


Multicast routing protocols come into play when a host needs to send the same message, or the same stream of data, to multiple destinations. Due to the unique characteristics of the mobile ad hoc networks such as host mobility, limited resources and very unreliable channel, traditional multicast protocols do not perform well in MANET scenarios. MANET multicast protocols should efficiently cope with dynamic topology changes such as fragile multicast tree structure. To compare and analyze multicast routing protocols, appropriate classification methods are important. Classification methods help researchers and designers to understand the distinct characteristics of different multicast routing protocols and find out the internal relationship among them. Therefore, we present protocol characteristics which are used to group and compare different approaches. These characteristics are mainly related to the information which is exploited for MANETs and the roles which nodes may take in the multicast routing process. There are several methods to classify multicast routing protocols for MANET. As reported in the literature, multicast routing protocols are broadly classified as tree-based and mesh-based protocols, based on the underlying structure of the multicast routes. The multicast routing protocols can be also classified as proactive and reactive protocols based on the route acquisition time, as sender-initiated and receiver-initiated protocols based on the multicast route initiation, and as source-based and group-shared protocols based on the forwarding state maintenance schemes. Some multicast routing protocols fall into one or more above mentioned categories. In the remaining of this section we briefly describe different classifications of multicast routing protocols.

\subsection{Based on Underlying Routing Structure}

One of the most popular methods to classify multicast routing protocols for MANETs is based on how distribution paths among group members are constructed (the underlying routing structure). According to this method, existing multicast routing approaches for MANETs can be divided into treebased multicast protocols, mesh-based multicast protocols and hybrid multicast protocols. In treebased multicast protocols, the members are connected through a tree structure. Tree-based protocols send the message along a tree-like data forwarding path which is rooted at the source of the multicast session, and supports a single path to each receiver. Depending on the number of trees per multicast group, tree-based multicast can be further classified as source-based multicast tree and group-shared multicast tree. Some of the tree-based multicast routing protocols are, bandwidth efficient multicast routing protocol (BEMRP) [12], multicast zone routing protocol (MZRP) [13], multicast core extraction distributed ad hoc routing protocol (MCEDAR) [14], differential destination-based multicast protocol (DDM) [15], ad hoc multicast routing protocol utilizing increasing id-numbers (AMRIS) [16], and ad hoc multicast routing protocol (AMRoute) [17].

Tree-based protocols may not perform well in the presence of highly mobile nodes because the multicast tree structure is fragile and needs to be readjusted frequently as the connectivity changes. Mesh-based multicast protocols have been proposed to address the problem by constructing a mesh structure with redundant links between mobile nodes. Indeed, mesh based protocols aims at enriching the connectivity among the multicast group members, and so they are more resilient against the network topology changes compared to tree-based protocols. Mesh based protocols generally suffer from the notorious broadcast storm problem, in which the flooding mechanism may result in excessive redundancy, contention, and collision. Furthermore, mesh-based protocols are more likely to form the routing loops. On-demand multicast routing protocol (ODMRP) [18], Clustered Group Multicast (CGM)[19], forwarding group multicast routing protocol (FGMP) [20], and core-assisted mesh protocol (CAMP) [21] are some of the existing mesh-based multicast protocols. Hybrid approaches like AMRout [17] are combinations of tree-based and mesh-based protocols to bring together the robustness of mesh-based multicast routing protocols and the low overhead of treebased protocols in a single scheme. 


\subsection{Based on Route Acquisition Time}

Based on when the multicast routes are determined, the multicast routing protocols fall into two different categories, namely proactive and reactive (on-demand). Proactive multicast routing continuously makes routing decisions so that multicast routes are immediately available when packets need to be transmitted. Proactive multicast routing protocols attempt to maintain consistent, up-to-date multicast routing information between every pair of multicast members in the network by propagating, proactively, route updates at fixed time intervals. As the resulting routing information is usually maintained in tables, the protocols are sometimes referred to as table-driven protocols. Ad hoc Multicast Routing protocol (AMRoute) [17] is a proactive multicast routing protocol. Reactive multicast routing protocols, on the other hand, establish a route to the multicast members only when there is a demand for it, usually initiated by the source node through route discovery process within the network. Once a route has been established, it is maintained by the node until either the destinations become inaccessible along every path from the source or until the route is no longer used or has expired. On-demand Location Aware Multicast (OLAM) [22], On-demand multicast routing protocol (ODMRP) [18], and On-demand Global Hosts for mobile Ad hoc Multicast services (OGHAM) [23] are some of the well known reactive multicast routing protocols. Proactive multicast routing protocols consume a great deal of radio resources to exchange routing information. Also, predetermined multicast routes may rapidly lose their validity in an ad hoc network because its topology frequently changes. Proactive multicast routing protocols generally provide better quality of service than reactive protocols. As in proactive protocols, routing information is constantly updated, routes to every destination are always available and up-to-date, and, hence, end-to-end delay can be minimized. For reactive protocols, the source node has to wait for the route to be discovered before communication can happen. This latency in route discovery might be intolerable for real-time communications.

\subsection{Based on Multicast Route Initiation}

The basic schemes for reliable multicast routing can be classified as sender-initiated and receiver- initiated approach based on who (sender or receiver) is responsible for route construction. A multicast routing protocol is termed as a sender-initiated protocol when the multicast tree construction is initiated by the multicast source. That is, Sender-initiated routing protocols establish a route only when a source node requests for a route. When the multicast members take the responsibility to connect to the source, then the protocol is termed as receiver-initiated multicast protocol.

In the sender-initiated approach, the sender is responsible for the error detection. A missing piece of data at a receiver is detected if the sender does not receive an ACK from the receiver. In this case, the need to retransmit a missing packet is handled by retransmitting the missing data from the source through a unicast. When several receivers have missing packets, the sender may decide to remulticast the missing packets to all receivers in the multicast group. When the sender-initiated approach is applied, only the sender (which keeps the history of multicast packets) is responsible for retransmitting the missing packet, and the corresponding retransmitting method is called senderoriented. Note that when the sender receives ACK signals from all the receivers, the corresponding packet can be removed from the history. Multicast Zone Routing (MZRP) [13] protocol, and Multicast Ad hoc on-demand Distance Vector Routing (MAODV) [24] protocol are two sender-initiated multicast routing protocols.

In the receiver-initiated approach, each receiver is responsible for error detection. Instead of acknowledging each multicast packet, each receiver sends a NACK once it detects a missing packet. If multicast packets are time stamped using a sequence number, a missing packet can be detected by a gap between sequence numbers of the receiving packets. WARM [25], short for Wireless Ad hoc RealTime Multicast, is a receiver initiated multicast routing protocol. 


\section{Multicast Routing Protocols}

In literature, the multicast routing protocols are mostly classified based on the underlying route structure. In such a classification, the multicast routing schemes are categorized as tree-based, meshbased, hybrid and stateless schemes. Tree-based schemes construct a multicast tree from each of the sources to all the receivers. Examples of protocols following this approach are BEMRP [12], MZRP [13], MCEDAR [14], DDM [15], AMRIS [16]. The main advantage of using a tree as the underlying forwarding structure is that the number of forwarding nodes tends to be reduced (although not optimized). However, a tree is very fragile when there is a high mobility in the network. Mesh-based approaches like ODMRP [18], CGM [19], FGMP [20], CAMP [21], by using additional links in their underlying forwarding structure, manage to deal with mobility very efficiently. The main drawback associated to the use of a mesh, is that the additional paths which are created can make an excessive consumption of network resources when sending data packets. Hybrid approaches like AMRout [17] try to combine the robustness of mesh-based ad hoc multicast routing and the low overhead of treebased schemes. Figure 1 shows the new classification of the multicast routing protocols presented in this paper. In the rest of this section, the existing multicast routing protocols are classified based on the underlying routing structure and described in more detail.

$$
\text { Multicast Routing Protocols }\left\{\begin{array}{l}
\text { Tree - Based }\left\{\begin{array}{l}
\text { Source - Based } \\
\text { Group - Shared } \\
\text { Mesh - Based } \\
\text { Hybrid } \\
\text { On Demand }
\end{array}\right. \\
\text { Stateless }
\end{array}\right.
$$

Figure 1. A new classification of multicast routing protocols for ad hoc networks

\subsection{Tree-based Multicast routing protocols}

In a tree-based multicast routing algorithm, a tree-like data forwarding path is constructed which is rooted at the source of the multicast session. The multicast tree is composed of a unique path from the multicast source to each of the multicast receivers. Tree-based protocols are highly efficient (efficiency is defined as ratio of the total number of data packets received by the nodes to the total number of data packet transmissions in the network) due to the absence of multiple redundant paths to the multicast source node. The main advantage of a tree as the underlying forwarding structure is that the number of forwarding nodes tends to be reduced (although not optimized). However, multicast trees form a virtual backbone which is fragile in ad hoc networks where the mobile hosts move freely anywhere. This is due to the fact that in a multicast tree there is no alternative path between the source and destination to tolerate the frequent network topology changes. Tree-based protocols primarily focus on how to construct the tree with the minimum control overhead, and involving the minimum cost. The cost metric is generally assumed the average distance of the multicast members from the multicast source. The control overhead involved in tree-based protocols is low, and the performance in terms of packet delivery ratio of such protocols, decreases as the mobility increases. The major difference between the tree-based and mesh-based multicast routing protocols lies in the manner in which a multicast message is relayed. In the tree-based multicast routing protocols, each intermediate node on the tree has a well-defined list of the next-hop nodes for a specific multicast session. It will send a copy of the received multicast message to only the 
neighboring nodes on its next-hop list. Figure 2 shows a sample tree-like multicast route to connect the multicast source to the multicast receivers.

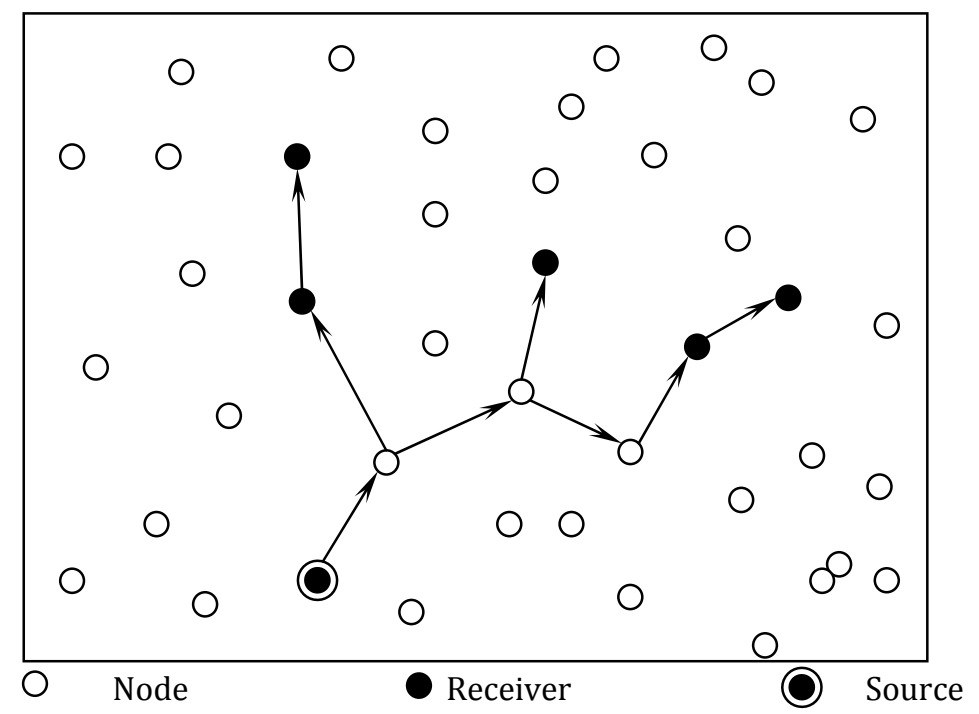

Figure 2. A Tree-based multicast route

Tree-based multicast routing protocols can be further subdivided into group-shared tree and source-based tree. In both cases, multicast trees are constructed to interconnect all the members of the multicast group. Data is delivered along the tree paths to reach all the group members. The source-based approach maintains, for each multicast source, an individual (minimal) tree towards all its multicast receivers. Source-based tree is separately established and maintained for each multicast source node of a multicast group. The advantage of source-based tree is that each multicast packet is forwarded along the most efficient (shortest) path from the source node to each multicast group member. However, this method incurs a lot of control overhead and cannot quickly adapt to the movements of the nodes in a mobile ad hoc network. Since the construction of a minimum cost tree (for each source) spanning all the members of the multicast group is expensive, some of the treebased multicast routing schemes use a (core-based) group-shared tree to distribute packets from all the sources. In the group-shared tree, a single tree is constructed for the whole group (e.g., regardless the sources location). Multicast packets are distributed along this shared tree to all members of the multicast group. Since the group-shared multicast tree only permits the multicast traffic to be sent out from the root to the multicast receivers, each source must forward its multicast traffic to the root. Multicast traffic of each source is then forwarded along the shared tree. The group-shared multicast tree is a well-known tree-based approach adopted by core based trees (CBT) [26]. A group-shared tree is a shortest-path tree rooted at some core node. The core node is also referred to as a center node or a rendezvous point. Core nodes may be chosen from some pre-selected set of nodes or some heuristics may be employed to select core nodes. Group-shared multicast tree is a more scalable approach than the source-based approach in which instead of building multiple trees for each multicast group, a single shared tree is used for all multicast source nodes. Representative groupshared multicast trees are built in MAODV [24] and AMRIS [16].

Source-based or group-shared, the optimal multicast tree is defined as a Steiner tree, although in some multicast routing protocols $[5,6]$, the minimum spanning tree problem (MST) is used to model the multicast routing problem. Constrained Steiner tree problem is shown to be NP-complete, and so 
several heuristics have been proposed to solve the multicast tree problem. The rest of this section briefly reviews the existing tree-based multicast routing protocols.

\subsubsection{Source-Based Multicast Tree}

Distance Vector Multicast Routing Protocol (DVMRP) [27] is a multicast routing protocol initially designed for wired networks. Modified version of DVMRP can be also applied for multicasting in wireless ad hoc networks. DVMRP builds and maintains source-based trees for sending multicast messages. The source-based tree is created by first flooding the whole network with the multicast traffic. After that the normal prune operations are conducted. In DVMRP, when a host receives a multicast packet, it sends the packet to all attached hosts and waits for a response. Hosts with no group members return a "prune" message, which eventually prevents further multicast messages for that group from reaching the host. The prune state is soft, that is, it will time-out within a set time interval. If after sending a prune and before the state can time-out, the host wants to join the group, it has to send a "graft" message upstream. DVMRP is inefficient when the number of receivers in the group is sparsely distributed. DVMRP builds its own routing table instead of reusing the existing unicast routing table for RPF, (short for reverse path forwarding) checking of incoming packets. A packet is assumed to have arrived on the RPF interface, if a host receives it on an interface that it uses to send unicast packets to the source. If the packet arrives on the RPF interface, then host forwards it out the interfaces that are present in the outgoing interface list of a multicast routing table entry. If it does not arrive on RPF interface, it is silently discarded to avoid loop-backs. The advantage of RPF is that it does not require the host to know about spanning trees. This way, multicast adapts automatically and only is sent where it is wanted [27].

Akbari Torkestani and Meybodi [96] proposed a link stability-based multicast routing protocol for wireless MANETs in which the multicast packets are forwarded along the Steiner tree links. The weight associated with a communication link is defined as its expected duration time which is assumed to be a random variable with unknown distribution. Expected link duration time is defined as the period of time during which the link is expected to be connected, and expected duration time of a multicast route is defined as the expected duration time of the weakest link. This protocol called LLMR aims at finding the most stable multicast route against the host mobility. LLMR is composed of a number of iterations and at each iteration a multicast route is constructed by finding a Steiner tree of the network topology graph. At each iteration, the selected multicast route is rewarded, if its expected duration time is longer than those seen so far and it is penalized otherwise. As the proposed algorithm proceeds, the choice probability of the most stable multicast route converges to one. Thyagarajan and Deering [28] proposed a hierarchical distance-vector multicast routing protocol. This approach involves partitioning the multicast backbone (MBone) into non-overlapping regions, while using DVMRP as the inter-region routing protocol. MBone is a virtual network developed to run on top of the physical Internet. Intra-region routing protocol may be accomplished by any of the multicast routing protocols. Jetcheva and Johnson [29] proposed an adaptive demand-driven multicast routing algorithm called ADMR that is able to adapt its behavior based on application sending pattern, to effectively detect the link breakage, and to terminate the routes that are no longer needed. ADMR creates and maintains source-based forwarding trees connecting each source with the receivers of the multicast group. The multicast forwarding state for a given multicast group and a source is conceptually represented as a loosely structured multicast-forwarding tree routed at the source. The forwarding mechanism is based on the shortest-delay path through the tree to the receiver members of the multicast group. In this method, packet forwarding is based on two types of flooding: tree flood and network flood. Tree flood occurs among nodes of the multicast tree, while network flood is flooding among all the nodes in the network. ADMR can detect the network mobility characteristics without requiring any global positioning system or additional control traffic. Depending on the results of such a mobility detection process, ADMR multicast source switch to the flooding for a period of time 
when the mobility is high, and attempt to operate efficiently with multicast again in the case of low mobility.

Associativity-based ad hoc multicast (ABAM) routing algorithm proposed by Toh et al. [30] establishes the multicast sessions using the association stability concept introduced in ABR unicast protocol [31] and requiring no underlying unicast routing protocol. ABAM constructs the sourcebased multicast trees using this concept on-demand, which reduces the communication overhead and improves end-to-end delay.

Zhu et al. [32] presented a heuristic algorithm called Bounded Shortest Multicast Algorithm (BSMA) for constructing the minimum-cost source-based multicast trees with delay constraints. BSMA starts with a Shortest Path Tree (SPT) to all destinations. It then iteratively replaces super-edges in the tree with lower cost paths until the total cost of the tree cannot be reduced any further. Although BSMA has a near optimal tree cost, it is not suitable for real networks due to its high computation cost. The proposed algorithm can set variable delay bounds on destinations and handles two variants of the network cost optimization goal: one minimizing the total cost of the tree, and another minimizing the maximal link cost. Instead of the single-pass tree construction approach used in most previous heuristics, the new algorithm is based on a feasible search optimization method which starts with the minimum-delay tree and monotonically decreases the cost by iterative improvement of the delaybounded tree.

Rouskas et al. [33] addressed the problem of constructing a source-based multicast tree with delay and delay jitter constraints for the one-to-many multicast paradigm and proposed a heuristic algorithm. Their algorithm can be also extended to accommodate the many-to-many. In the proposed algorithm, for each multicast source, a source-based multicast tree is initially constructed. Then, a minimum set of multicast trees is found such that for each multicast source there exists at least one feasible multicast tree.

In [34], Wang et al. presented a distributed algorithm called L-REMiT for enhancing the lifetime of a source-based multicast tree in wireless ad hoc networks. In this paper, the lifetime of a multicast tree is defined as the duration from the formation of the tree to the time when the first node fails due to battery energy exhaustion. The proposed multicasting algorithm assumes that the energy consumed to forward a packet is proportional to the forwarding distance and channel characteristics, and that wireless nodes can dynamically adjust their transmission power.

Protocol independent Multicast (PIM) operates in two modes: dense mode (PIM-DM) and sparse mode (PIM-SM). PIM-DM [35] proposed by Deering et al. operates similar to DVMRP. PIM-dense mode is designed for multicast communication in small networks and high group density. No rendezvous point (RP) is used. The protocol builds a source-based multicast tree for each sender as soon as it starts sending data. Multicast routing entries are kept in related routers under the mechanism of timeout. At startup, it assumes that all subnets wish to receive data. Therefore, flooding and pruning are used. Graft data units are sent to support immediate integration of new group members into the multicast tree. Due to flooding, data can end up being sent unnecessarily to network areas in which no group members are located. However, this is considered acceptable because it is assumed that the density of a group will be very high, the distance between members are short, and consequently the additional overhead will be low .

Moy [36] proposed an extension of OSPF unicast routing protocol called Multicast Open Shortest Path First (MOSPF) for multicast routing. MOSPF knows all the multicast groups currently residing in the network and builds an individual distribution tree for each source/group pair, i.e., it constructs source-based multicast trees. Distribution trees must be re-computed either periodically or when there is a link state change. MOSPF is a link state routing protocol that builds the map of the network topology, including location of domains and tunnels. It selects the best path to the required receivers using Djikstra's shortest path algorithm. When there are multiple sources or many groups, it is CPU intensive. It is best used when relatively few sources or groups are active at any given time. It does not work well in presence of unstable links, as it leads to frequent state update and the associated 
computations. MOSPF does not support tunneling. The path is calculated only on-demand and cached for later use.

\subsubsection{Group-Shared Multicast Tree}

Multicast Ad hoc on-demand Distance Vector Routing protocol so-called MAODV proposed by Royer and Perkins [24] maintains a shared tree for each multicast group, consisting of only receivers and relays. MAODV results from the well-known unicast routing algorithm called AODV proposed by the same authors [37]. MAODV is an on-demand routing protocol that discovers the route only when a node has data to send. It is a hard state protocol, i.e., if a member node of a multicast group desires to terminate its group membership, it must request for termination. When the core revokes its own group membership, it selects one of its tree neighbors to become the new core. If the new core is not a group member, one of its downstream tree neighbors is selected to become the new core. This core selection process is continued until a group member is found to become the new core. When a mobile node wants to join a multicast group or send a message but does not have a route to the group, a Route Request (RREQ) is originated. All the nodes that are members of a multicast group together with the nodes that are not members of the group but their position are very critical for forwarding the multicast information, compose the tree structure. Every multicast group is identified by a unique address and group sequence numbers for tracing the freshness of the group situation. In MAODV, a node maintains multicast routing table for the group tree structure. This table contains the multicast group address, the multicast group leader address, the multicast group sequence number, hop count to the multicast group leader, next hop information and the lifetime. Nodes in a tree structure are described as downstream and upstream nodes. A downstream node is a neighborhood node, which is further from the group leader (more hop counts from the group leader). An upstream node is a neighborhood node which is nearer to the group leader (less hop counts from the group leader). It is obvious that a group leader has only downstream nodes. Whenever a node leaves the multicast group, the tree structure is pruned. If an edge in the multicast tree is broken, the downstream node of the edge is responsible for finding a path to the core and reforming the multicast tree. If this node cannot find a path to the core within a certain time period, the network is assumed to be disconnected and the subtree rooted at this node becomes another multicast tree. If it is a group member, this node then becomes the core of the newly formed multicast tree; otherwise, the core selection process is used to find the core of the newly formed multicast tree. If the network becomes connected later, the multicast trees of the same group are merged into one whose core is the same as that of the multicast tree having the largest address.

In [38], Gupta and Srimani proposed a method similar to MAODV, where the core migrates to the group member median located at the tree centroid. They presented distributed core selection and migration algorithms for mobile ad hoc networks with dynamically changing network topology. The proposed core location method is based on the notion of median node of the current multicast tree instead of the median node of the entire network. The rationale is that the mobile ad hoc network graphs are in general sparse, and so the multicast tree is a good approximation of the entire network for the current purpose. The adaptive distributed core selection and migration method uses the fact that the median of a tree is equivalent to the centroid of that tree. This leads to a significant efficiency improvement since the median (or the centroid) computation in a tree does not need distance information.

Liu et al. [39] proposed a heuristic method to reduce the cost of the core-based group-shared multicast tree by minimizing the number of non-leaves in the multicast tree, where the cost is evaluated by the total bandwidth consumption of multicasting packets among all group members. They introduced a distributed heuristic in which the multicast tree is dynamically constructed and adjusted rather than finding a new multicast tree. To do so, several non-leave nodes are transferred into leaves in an existing multicast tree. 
In [16], Wu and Tay presented a multicast protocol called AMRIS, short for Ad hoc Multicast Routing protocol utilizing Increasing ID numbers. AMRIS is an on-demand (reactive) multicast routing protocol which constructs a shared delivery tree to support multiple senders and receivers within a multicast session. The conceptual idea behind AMRIS is to assign every node in a multicast session an ID number. A delivery tree rooted at a particular node called Sid joins up the nodes participating in the multicast session. The relationship between the ID numbers (and the node that owns them) and Sid is that the ID numbers increase in numerical value as they radiate from the root of the delivery tree. The significance of the Sid is that it has the smallest ID number within that multicast session. Utilizing the ID numbers, nodes are able to adapt rapidly to changes in link connectivity. Recovery messages due to link breakages are confined to the region where the breakage occurred.

The Zone Routing Protocol (ZRP) [40] is a hybrid unicast protocol that proactively maintains routing information for a local neighborhood (routing zone), while reactively acquiring routes to destinations beyond the routing zone. It is composed of IntrAzone Routing Protocol (IARP) [41], IntErzone Routing Protocol (IERP) [42] and Bordercast Resolution Protocol (BRP) [43] with a querycontrol mechanism. Zhang and Jacob [13] extended ZRP for application to multicast routing and call it the Multicast Zone Routing Protocol (MZRP). MZRP is a shared tree multicast routing protocol that proactively maintains the multicast tree membership for nodes' local routing zones at each node while establishing multicast trees on-demand. It is scalable to a large number of multicast senders and groups. In MZRP, IP tunnel mechanism is employed to improve the data packet delivery ratio during the transmission.

Canourgues et al. [44] proposed a shared tree multicast routing scheme called STAMP for large wireless mobile ad hoc networks. The proposed scheme clusters the network to relieve the scalability constraints. STAMP is a core-based shared tree multicast routing protocol independent from the underlying unicast routing protocol. Compared to other shared-tree multicast routing protocols, STAMP achieves noticeably better performance even under mobility conditions since it takes advantage of the broadcast capacity of the medium to deliver data on the tree.

A multicast routing protocol for mobile Ad hoc network should find a compromise between the routing overhead and data transmission efficiency so that it can efficiently use bandwidth and power. For this aim, Kaliaperumal et al. [45] proposed a reactive (on-demand) multicast routing protocol called ACMP, short for Adaptive Core based Multicast Routing protocol, which constructs and maintains a group-shared tree using adaptively selected core only when the group traffic exists. The proposed multicast routing scheme attempts to react more quickly to the broken tree edge by detecting link failures during data forwarding process. In this method, once a link failure is detected, ACMP uses local route recovery to establish a temporary route and periodical tree refreshing to maintain an optimal multicast tree.

Estrin et al. [46] proposed another version of PIM called PIM-SM (short for Protocol Independent Multicast-Sparse Mode) in which a node is selected as the RP and all group communication takes places by sending the packets to it. Each of the sources in a PIM-SM multicast group sends its packets to the RP. Since it builds unidirectional shared tree, only the RP can forward data to the members. Intermediate nodes should forward the data only to the RP. All PIM-SM traffic is transported by unicast instead of multicast. The PIM-SM router with highest IP address is Designated Router (DR) for the subnet and is responsible for sending Prune/Join messages to the RP. DR determines the RP for a group using a hash function. The tree obtained is not necessarily optimal. PIM-SM allows switching of the receiver connectivity to the tree from shared-tree path to the source-based tree path. When a group has numerous highly active sources, the bandwidth of the shared links may not be able to accommodate all the traffic. The QoS requirements of a user may not be met along the shared-tree, thus it has to switch to its shortest path to the RP. When a receiver switches from shared-tree mode to source-based tree mode, the number of packets dropped during the transition period is dependent on two factors: The delay difference between the shared-tree path and source-based tree path from the source to the receiver and the sending rate of the source. 
Ballardie et al. [26] proposed a core based multicast routing algorithm called CBT (Core Based Tree) in which a single shared multicast tree is used to connect all the different multicast groups and their members. CBT builds a single bidirectional shared tree for data transmission. CBT involves having a single node, known as the core of the tree, from which branches emanate. These branches are made up of the other nodes, so-called non-core nodes, which form a shortest path between a member host and core. The core need not be topologically centered between the nodes on the tree, since multicasts vary in nature, and so can the form of a core-based tree. CBT involves having a single core tree per group, with additional cores to add an element of robustness to the model. Core selection is one of the major issues in CBT and can be handled by the various heuristics proposed for core selection. When an intermediate node receives a packet, it forwards it to the remaining members of the group that are downstream to the node. It does not need to forward it to the core. In [47], Gupta and Srimani proposed a core-based shared tree for reliable multicasting in multi-hop mobile radio networks. The proposed protocol is reliable, i.e., it guarantees message delivery to all the multicast members even when the topology of the network changes during the multicasting. They introduced a notion of the forwarding region which is used to limit the scope of the flooding messages only to the vicinity of the nodes which witness topology change due to host mobility. This notion is used to glue together the fragments of the multicast trees. The proposed protocol is more efficient in conserving network bandwidth, especially when the multicast groups are small in size and the multicast group members are localized to a certain region of the network.

Since the constrained multicast routing problem is known to be NP-complete, many proposed approaches are based on heuristic methods, such as ant colony algorithms, genetic algorithms and fuzzy-based algorithms. The rest of this section reviews some of the existing heuristic methods.

\section{Ant Colony Approach}

Ant colony optimization (ACO) is a population-based meta-heuristic that can be used for finding approximate solutions to difficult optimization problems [48]. ACO studies artificial systems that take inspiration from the behavior of real ant colonies and which are used to solve discrete optimization problems. In ACO, a set of software agents called artificial ants search for good solutions to a given optimization problem. To apply ACO, the optimization problem is transformed into the problem of finding the best path on a weighted graph. The artificial ants (hereafter ants) incrementally build solutions by moving on the graph. The solution construction process is stochastic and is biased by a pheromone model, that is, a set of parameters associated with the graph components (either nodes or edges) whose values are modified at runtime by the ants $[48,49,50]$.

Liu et al. [51] proposed a novel scheme based on ACO called DMACO for finding the minimum cost multicast tree with delay constraint, which is an NP-complete problem. The proposed algorithm is a fully distributed routing scheme which does not need a core node to possess the whole network information. In this method, the ants only use the local information to finish the task of finding the destinations and establishing the multicast tree. The proposed scheme is easy to realize by three types of ants with simple structures: forward ants, backward ants, and pheromone update ants. A forward ant is one that the source sends to look for the destinations. A forward ant will be killed when it reaches the destination, and a backward ant is generated and returned to the source node along the inverse path. When all ants find all the destinations and construct the corresponding multicast trees, the source node will send pheromone update ants to update the pheromone table of corresponding nodes. The proposed scheme is loop free and has small overhead compared with the flooding method. In [52], Wang and Xie combined an improved version of ant colony optimization with a heuristic algorithm to solve the multicast routing problem. In this paper, the multicast routing problem is considered as the Steiner tree problem, and to solve this problem the proposed algorithm attempts to find the minimum number of Steiner nodes that are not included in the multicast routing group. A modified ant colony system is presented by Zhang et al. [53] to solve the single-origin-multi- 
destination routing problem with no constraints. This algorithm first transforms the connective network into the corresponding distance complete graph, and then develops a Prim's-algorithm-like route construction process with an inclination factor towards the multicast vertices. Tseng et al. [54] proposed an ACO-based algorithm for solving the degree-delay-constrained minimum spanning tree (DDCMST) problem. DDCMST consists of three mechanisms: the spanning tree construction, the local updating rule, and the global updating rule, stated as follows. Spanning tree construction is a fundamental mechanism to solving the DDCMST problem by evolutionary algorithms. It introduces APrim as a modified version of Prim's algorithm (Prim, 1957). APrim starts tree-constructing from a source node, and then iteratively selects a new edge from the set of candidate edges to expand the tree. Edges are selected based on a revised pseudo-random-proportional rule. After an ant has found a spanning tree, the pheromone levels on the edges of that spanning tree are updated using a local updating rule. The proposed algorithm repeatedly constructs spanning tree and updates pheromones locally and globally until the stop condition is met.

\section{GENETIC Algorithm APPROACH}

Genetic algorithms are a particular class of evolutionary algorithms in which the techniques inspired by the evolutionary biology such as inheritance, mutation, selection, and crossover are used. Genetic algorithms are categorized as global search heuristics. A typical genetic algorithm requires a genetic representation of the solution domain, as well as a fitness function to evaluate the solution domain. The fitness function is defined over the genetic representation and measures the quality of the represented solution. The fitness function is always problem dependent. Once we have the genetic representation and the fitness function defined, genetic algorithm proceeds to initialize a population of solutions randomly. Then, it improves the population through repetitive application of genetic operations. In [55], Zhang and Leung proposed an orthogonal genetic algorithm for multimedia multicast routing. Its salient feature is to incorporate an experimental design method called orthogonal design into the crossover operation. The salient feature of the proposed genetic algorithm is to incorporate an experimental design method called orthogonal array into the crossover operation, so that the resulting operation can sample the genes from $n$ parents in a statistically sound manner to produce $\mathrm{j}$ offspring. As a result, the orthogonal genetic algorithm can search the solution space in a statistically sound manner and it is well suited for parallel implementation and execution. In general, a larger orthogonal array gives a better solution quality but has a higher computational complexity. They execute the orthogonal genetic algorithm to solve two sets of benchmark test problems. The results indicated that for practical problem sizes, the orthogonal genetic algorithm can find nearoptimal solutions within moderate numbers of generations. Atzori and Raccis [56] proposed a genetic algorithm which focuses on the problem of network capacity assignment for multicast services. The problem is firstly formalized by defining a cost function to evaluate the goodness of a given capacity allocation configuration. Then, a novel approach based on the genetic algorithms is provided to find the near-optimal solution. The genetic algorithm proposed to solve the multicast capacity assignment problem relies on two phases. The first is the search of a set of tree solutions for each multicast session alone, and the second phase is the search of the best trees combination for all the multicast sessions together starting from the output of the first phase. A novel heuristic multicast routing algorithm was proposed by Hwang et al. [57] based on genetic algorithms. The new multicast routing algorithm tries to minimize the multicast cost while maintaining a reasonable path delay. The number of generations required to reach a good solution is significantly reduced by preferring shorter routes in initializing the chromosome pool and reusing the past solutions as the initial chromosomes for the new search. To apply this algorithm to real networks, they also propose several methods for reducing the computational complexity. The main advantages of the proposed genetic algorithms are as follows. Since the solutions are coded as bit strings, referred to as chromosomes, large problems can be easily handled by using long strings. Genetic operations, such as crossover and mutation, are very easy to 
implement. With a pool of chromosomes (candidate solutions), genetic algorithms search the solution space at different corners in parallel, and so the algorithm can be easily implemented on multiprocessor machines to search in parallel. Randomized genetic operations, such as mutation, can keep the search from being trapped by local-optima.

Chiang et al. [58] proposed a new and efficient spanning tree encoding scheme based on genetic algorithms for solving the multicast routing problem in mobile ad hoc networks. They also proposed three genetic-based operation tailored for the new encoding scheme. Topology crossover, node mutation, and edge mutation are three operations proposed in this paper. The proposed method uses the encoding scheme proposed by Huang et al. [59] called sequence and topology encoding (ST encoding). Topology crossover performs conventional one-point crossover on the topology chromosomes of two spanning tree codes to form new topologies. The idea behind edge mutation is to cut down a randomly selected subtree, and then grafts it to a randomly selected node in the remaining tree to form a new tree. Since a chromosome is a permutation of all the tree nodes, node mutation randomly selects two genes from a chromosome except the root and swaps them. An energy-efficient genetic algorithm was proposed by Yen et al. [60] to resolve the multicast routing problem with multiple QoS constraints in mobile ad hoc networks. Furthermore, they designed a source-tree-based routing algorithm and built the shortest-path multicast tree to minimize delay time by using a small population size in the genetic algorithm. In this algorithm, only a few nodes are involved in the route computation. They also improved the genetic sequence and topology encoding and prolonged the lifetime of the mobile nodes which are responsible for calculating the residual energy of all nodes in a multicast tree. Zahrani et al. [61] introduced a genetic local search heuristic that utilizes logarithmic simulated annealing in a pre-processing step for an analysis of the landscape generated by a multicast routing problem and the associated objective function. The annealing procedure allows the algorithm to estimate the depth of the deepest local minima. The genetic local search employs the partially mixed crossover (PMX) operation in-between sequences of downward and upward search steps, where the elitist model is applied. The PMX operation seems to be particularly suited to problems like multicast routing, since the outcome of the operation is always defined for two given parent routing orders.

\section{FUZZY LOGIC APPROACH}

In 1965, Lotfi Zadeh [62] proposed fuzzy set theory and later established fuzzy logic based on the fuzzy sets. Fuzzy logic is a form of multi-valued logic derived from fuzzy set theory to deal with reasoning that is approximate rather than precise. In contrast with binary sets having binary logic, also known as crisp logic, the fuzzy logic variables may have a membership value of not only 0 or 1 . Just as in fuzzy set theory with fuzzy logic the set membership values can range (inclusively) between 0 and 1 , in fuzzy logic the degree of truth of a statement can range between 0 and 1 and is not constrained to the two truth values \{true (1), false (0)\} as in classic propositional logic. And when linguistic variables are used, these degrees may be managed by specific functions. Fuzzy logic has been applied to diverse fields, from control theory to artificial intelligence. Oh et al. [63] proposed a fuzzy-based multicast routing algorithm that provides a linguistic guarantee for disruption free service despite handoffs occurred during an active connection. The fuzzy-based multicast scheme estimates the velocity and the direction of a mobile host. Then, the fuzzy multicast determines the basic fuzzy set for each neighboring host using the membership function, composes the multicast group for the mobile host using the control rules, and computes a multicast time using the defuzzifier of linguistic guarantee for disruption free service. Then, according to the specified linguistic guarantee for a user, data packets are transmitted to the forecasted neighbors where the mobile host will be handoff potentially, after the staggered time. Chiang and Huang [64] proposed a new structured representation of the multicast routing problem and improved it by using the fuzzy Petri nets with the concept of immediately reachable set. They also proposed a Fuzzy Reasoning Algorithm for finding the 
multicast tree and improving the efficiency of the routing scheme in ad hoc networks. A Fuzzy Logicbased secure multicast routing protocol called (FLSL) is proposed by Nie et al. [65] for mobile ad hoc networks. The basic idea of FLSL is to utilize the local multicast mechanism and the security-level to select the securest route. The proposed algorithm of Security-Level is an adaptive fuzzy logic-based algorithm that can adapt itself with the dynamic conditions of the mobile hosts. An interesting property is that every node in the MANET has the field of Security-Level based on the fuzzy logic in the route tables to select the highest Security-Level route. The FLSL routing protocol can improve MANET's security. It is feasible to the weak security character of mobile ad hoc networks. Su et al. [66] proposed the fuzzy modified AODV multicast routing protocol called FMAR to select two comparably stable routes by computing dynamic route lifetime for multicast routing or layered video streaming. The model compares and ranks different route lifetimes by the weighted Multi-criteria which are based on hop count, number of control packets and energy limitation. The reason for using the fuzzy logic is the uncertainties associated with node mobility and the estimation of link crash. In this protocol, the packets are multicast via multiple paths to multiple receivers of a multicast group instead of successively performing single cast in ad hoc networks.

\subsection{Mesh-based Multicast Routing Protocols}

In a mesh-based multicast routing protocol, multiple routes may exist between any pair of source and destination, which is intended to enrich the connectivity among group members for better resilience against topology changes. In a mesh-based multicast routing protocol, packets are distributed along the mesh structures that are a set of interconnected nodes. Route discovery and mesh building are accomplished in two ways: by using broadcasting to discover routes or by using core or central points for mesh building. Mesh-based protocols perform better in high mobility situation as they provide redundant paths from source to destinations while forwarding data packets. However, mesh-based approaches sacrifice multicast efficiency in comparison to tree-based approach. Mesh-based protocols have high packet delivery ratios compared to tree-based protocols, but incur more control overhead in route maintenance and redundant transmission. As noted before, the major difference between the tree-based and mesh-based multicast routing protocols lies in the manner in which a multicast message is relayed. In most of the mesh-based multicast routing protocols, however, relaying transmission takes a more redundant approach: each node on the mesh will rebroadcast the message upon its first reception of the message. As shown in Figure 3(a), the network-wide broadcasting (or global flooding) brings about the broadcast storm problem that occupies the network bandwidth and consumes the node energy which are scarce resources in MANET. Scoped flooding (or limited scope flooding) is an appealing solution to this problem. In this method, the network nodes along the path connecting the multicast source to the multicast receivers are only responsible for rebroadcasting the multicast messages. This significantly reduces the message overhead. Figures 3(a) and 3(b) show the global flooding and scoped flooding in a sample network having 11 nodes and 3 multicast receivers, respectively. From these figures, it can be seen that all the network nodes are involved in the global flooding mechanism, while in scoped flooding only two forwarding nodes toward the destinations rebroadcast the messages and the others only hear to the channel.
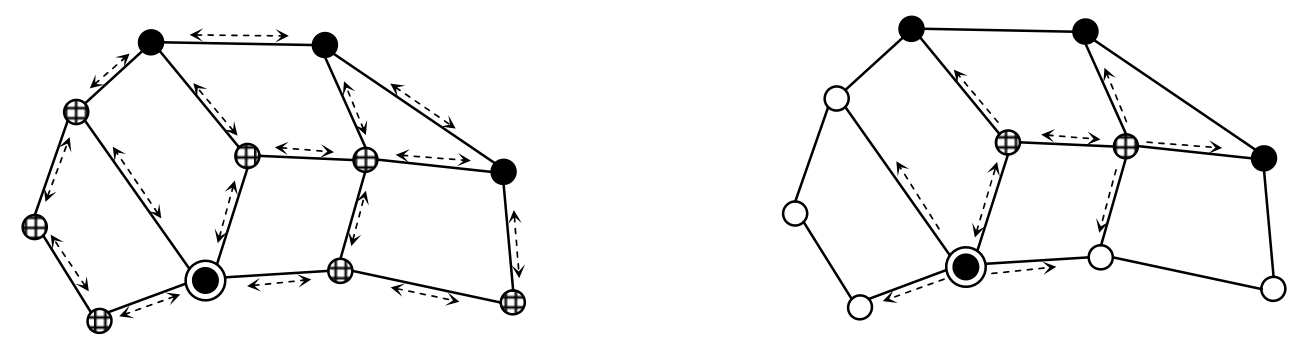


\section{O Network Node $\oplus$ Forwarding Node $\bigcirc$ Receiver $\bigcirc$ Source

(a)

Figure 3. (a)Global flooding, (b)Scoped flooding

Lee et al. [18] designed an on-demand multicast routing protocol called ODMRP for mobile ad hoc networks. ODMRP applies on-demand routing techniques to avoid channel overhead and improve scalability. It uses the concept of forwarding group [20], a set of nodes which is responsible for forwarding multicast data on the shortest paths between any member pairs to build a forwarding mesh for each multicast group. By maintaining and using a mesh, ODMRP avoids drawbacks of multicast trees in mobile wireless networks, such as intermittent connectivity, traffic concentration, frequent tree reconfiguration, non-shortest path in a shared tree. Indeed, ODMRP uses a forwarding mesh for data distribution. ODMRP is a reactive (on-demand) protocol that delivers packets to destination(s) on a mesh topology using scoped flooding of data within the mesh. As an on-demand routing mechanism, ODMRP reduces unnecessary channel overhead. On the other side, since routes are setup on demand, delay increases. The created mesh sub-network provides richer connectivity among multicast members compared to trees. Hence, unlike trees, frequent reconfigurations are not required. ODMRP takes a soft-state approach to maintain multicast group members. No explicit control message transmission is required to leave the group. ODMRP establishes and maintains group membership and multicast routes by the source on demand. The major strengths of ODMRP are its simplicity and scalability.

PatchODMRP [67] is one of the first derivatives of ODMRP. It uses the same procedure to construct the initial forwarding mesh structure, but it differs from ODMRP in that it take local repairing approach on detection of mesh destruction (i.e. link breakage) to avoid frequent mesh reconstructions. PoolODMRP [68] enhances route repair cost of PatchODMRP using so-called pool nodes. The pool nodes are defined as the neighbor nodes of forwarding nodes and they collect route information by overhearing data transmission. PoolODMRP reduces local route repair overhead by using the pool nodes. Cai et al. [69] improved the performance of PoolODMRP by using a passive acknowledge scheme so as to reduce the overhead more. The new scheme is called PDAODMRP, short for Passive Data Acknowledge ODMRP. It removes MAC layer BEACON signal and detects a route breakage by the passive acknowledge while data transmitting. Due to the use of the passive acknowledge scheme, PDAODMRP local route recovery is initiated by a upstream node whereas a downstream node starts a local recovery in previous two protocols. An enhanced version of ODMRP called E-ODMRP was proposed by Oh et al. [70]. The efficiency, simplicity, and robustness of ODMRP render it one of the most widely used MANET multicast protocols. The main reason for robustness of ODMRP is the periodic route refreshing. ODMRP rebuilds the data forwarding mesh on a fixed interval and thus the route refresh interval is a key parameter that has critical impact on the network performance. If the route refresh rate is too high, the network will undergo too much routing overhead, wasting valuable resources. If it is too low, ODMRP cannot keep up with network dynamics. E-ODMRP with the refresh rate dynamically adapted to the environment adjusts the refresh interval based on the route lifetime such that the forwarding mesh is refreshed before it breaks. The Performance Enhanced On-Demand Multicast Routing Protocol (PEODMRP) [71], and the On-Demand Multicast Routing Protocol with Multipoint Relay (ODMRP-MPR) [72] are some other ODMRP-based multicast protocols. PEODMRP reduces control packet overhead via limiting the transmission area of Join Query flooding. If forwarders are set within a time, it responds with Join Reply without Join Query relay. PEODMRP has advantage when multiple sources exist in the same multicast group. ODMRPMPR minimizes the broadcasting overhead by reducing duplicated packet-forwarding. Only designated one hop neighbor nodes relay packets while packet transmitting. It significant reduces duplicated packet transmission but packet delivery ratio decreases in high mobility network. 
Core-Assisted Mesh Protocol (CAMP) [73] is a proactive mesh-based multicast routing protocol, which establishes a multicast mesh for each multicast group. CAMP avoids the need for network-wide floods (or global flooding) from each source to maintain multicast meshes by using one or more cores per multicast group. A receiver-initiated approach is used for receivers to join a multicast group by sending unicast join requests towards a core of the desired group. To join a multicast group, a node first checks if any of its neighbors already is a member of the multicast mesh. If that is the case, the node announces a membership request to its neighboring nodes. If there are no nodes to belong the mesh, node sends a join message to one of the core node. The core nodes are not necessarily needed. Because if there is no available core nodes, an expanded ring search is used to find at least one node to belong the mesh. The drawbacks of CAMP is that it needs the pre-assignment of cores to groups and a unicast routing protocol to maintain routing information about the cores, and this may incur considerable overhead in a large ad hoc network. Unlike CBT [26], in which all traffic flows through the core nodes, the core nodes in CAMP are used only to limit the control traffic when the receivers are joining multicast groups.

Lin and Chao [19] put forward a new protocol for multicasting in multihop wireless networks called clustered group multicast (CGM). In CGM, there is a set of forwarding nodes (called multicast virtual backbone) which are responsible for forwarding the multicast packets to the receivers. Unlike the multicasting protocols in wired networks (e.g. Internet) which construct and maintain a shortest path tree for every multicast (source, group) pair, the connectivity among the nodes in the backbone is of no longer importance. Thus, there is no tree maintenance overhead. In addition, a key feature of CGM is the use of the advertising agents to reduce advertising traffic to the system. An advertising agent acts as both a server and a client for the purpose of advertising join requests on behalf of its local clients. Because in CGM multicast traffic is only allowed to be delivered over the backbone, CGM restricts the amount of hosts participating in the backbone to decrease the impact of multicast traffic to the system.

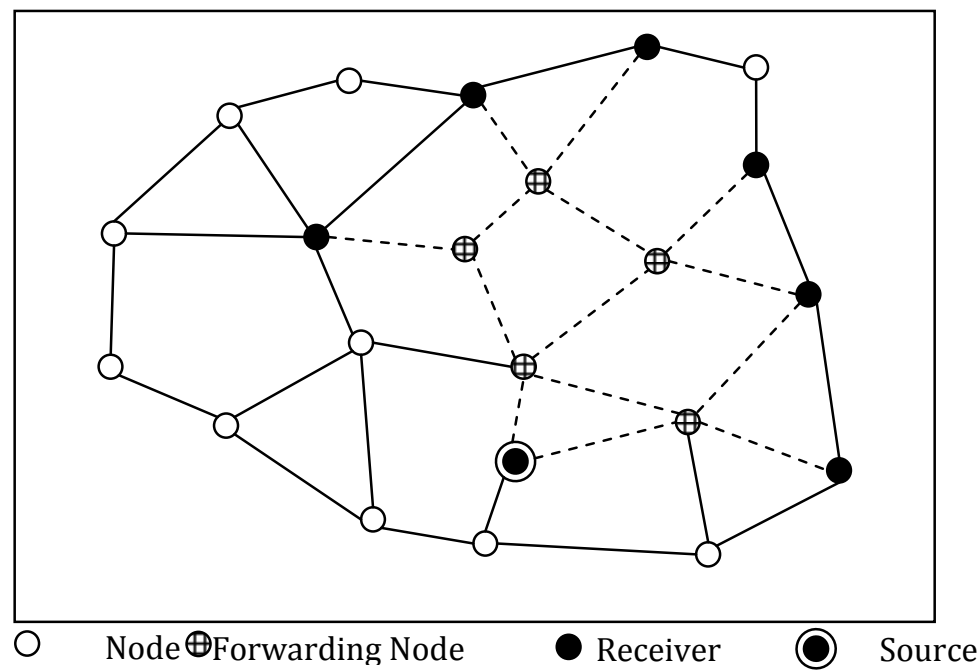

Figure 4. A mesh-based multicast route

In [20], Chiang et al., proposed another multicast routing protocol called Forwarding Group Multicast Protocol (FGMP) tailored for highly mobile ad hoc environments. FGMP is another meshbased routing protocol, where the mesh of nodes is formed and maintained by either receivers (FGMP-RA) or senders (FGMP-SA). To manage the maintenance overhead, FGMP-RA is used to form 
the mesh of nodes if the number of receivers is smaller than the number of sources. FGMP-SA is used otherwise. Like ODMRP, FGMP uses the network-wide flooding to build the mesh forwarding structure. FGMP associates a forwarding group (FG) with each multicast group. Any node in forwarding group is in charge of forwarding (broadcasting) the multicast packets. In other words, when a forwarding node receives a multicast packet, it will broadcast the packet if it is not a duplicate. All neighbors can hear it, but only the neighbors that are in FG will broadcast it, if they have not broadcasted it so far. The major problem of FGMP is how to elect and maintain the set FG of forwarding nodes. The size of FG should be as small as possible to reduce the wireless channel overhead, and the forwarding path from senders to receivers should be as short as possible to get the high throughput. This scheme can be viewed as a limited scope flooding. That is, flooding is contained within a properly selected forwarding set (mesh). It is interesting to note that with the proper selection of the forwarding group, FGMP scheme can emulate the behavior of any of the existing schemes. For example, to produce the global flooding, the FG must include all nodes in the network. For CBT, the FG is restricted to the nodes on the shared tree except the leaf nodes, and in DVMRP, FG includes all the non-leaf nodes on the source trees. Figure 4 shows a mesh-based multicast route connecting the multicast source to a set of multicast receivers having six members through a group consisting of five forwarding nodes. From Figure 4, it can be seen that since only the forwarding nodes are responsible for relaying the multicast packets, FG must cover all multicast members.

The simplest method for mesh-based multicast routing is to flood (network-wide broadcast) the multicast messages, which not only wastes the scarce resources of wireless hosts, but also diminishes the throughput of the network. This method suffers from the notorious broadcast storm problem which results in excessive redundancy, contention, and collision. One way to avoid the flooding is to use the concept of the dominating set (DS). The main idea behind the dominating set is very similar to that of forwarding group. Indeed, the aim of using the dominating set is to design an intelligent and scalable flooding scheme via limiting the flooding scope. A set is dominating if all the nodes in the network are either in the set or neighbors of nodes in the set. A connected dominating set (CDS) extracts a virtual backbone of the entire network through which the messages can be routed. Virtual backbone formation is a promising approach for multicast routing in which the routing overhead can be minimized, where the number of hosts responsible for the route discovery and data transmission can be reduced to the number of hosts in the backbone.

In [14], Sinha et al. proposed a multicast routing algorithm called Multicast Core Extraction Distributed Ad hoc Routing (MCEDAR) for wireless ad hoc networks. The proposed protocol bases its core-extraction criteria on the dominating set concept for the network topology, namely, a node is either a core or an immediate neighbor of a core. Besides the adopted virtual backbone procedures, the protocol itself has four key components: the mesh-based multicast structure, the join protocol, the core (broadcast-based) forwarding protocol, and the leave pruning and reconstruction protocols. In MCEDAR, a distributed minimum dominating set algorithm is applied for backbone formation purpose, and the resulting backbone has the property that all nodes are within one hop away from a core node. A core node and its dominated node set form a cluster. Once a virtual backbone is formed, the multicast operation is divided into two levels, the lower level multicasting which is within the cluster and upper level multicasting which is within the backbone.

Finding the minimum CDS problem in an arbitrary graph is a NP-Hard problem [74, 75], and so a host of approximation algorithms have been proposed to find a near optimal solution to this problem in a reasonable time. Guha and Khuller [76] proposed two centralized greedy heuristic algorithms with bounded performance guarantees for connected dominating set formation. In the first algorithm, the connected dominating set is grown from one node outward, and in the second algorithm, a weakly connected dominating set is constructed, and then the intermediate nodes are selected to create a CDS. Guha and Khuller [76] also proposed an approximation algorithm to solve the Steiner CDS problem, in which only a specified subset of vertices has to be dominated by a CDS. Butenko et al. [77] also proposed a prune-based heuristic algorithm for constructing the small connected dominating 
sets. In this algorithm, the connected dominating set is initialized to the vertex set of the graph and each node is then examined to determine whether it should be removed or retained. If removing a given node disconnects the induced sub graph of the connected dominating set, then it is retained and otherwise removed. The algorithm proposed by $\mathrm{Wu}$ and $\mathrm{Li}$ [78] first finds a CDS and then prunes certain redundant nodes from the CDS. Alzoubi et al. $[79,80]$ proposed two distributed heuristic algorithms for constructing CDS in a wireless ad hoc network. These algorithms first employ the distributed leader election algorithm [79] to construct a rooted spanning tree from the original network topology. Then, an iterative labelling strategy is used to classify the nodes in the tree to be either dominator or dominatee, based on their ranks. The first heuristic uses the ID-based approach for ranking the nodes, and the second heuristic uses the level-based approach. Alzoubi et al. [81] proposed another distributed algorithm for approximating the minimum CDS in a wireless ad hoc network. The proposed algorithm first constructs a maximum independent set (MIS), and then forms the CDS by adding more intermediate nodes into the MIS.

Li et al. [82] proposed a greedy MIS-based algorithm for constructing CDS in wireless networks. The first step of the proposed algorithm focuses on the forming a MIS of the network topology graph. At the second step, a greedy approximation is employed for finding a Steiner tree with the minimum number of Steiner nodes to interconnect the nodes in the MIS. Gao et al. [83] also presented a new distributed approximation algorithm that constructs a MCDS for wireless ad hoc networks based on a maximal independent set (MIS). In the proposed algorithm, each node only requires the knowledge of its one-hop neighbors and there is only one shortest path connecting two dominators that are at most three hops away. Xie et al. [84] proposed a novel distributed approximation algorithm to construct the MCDS in wireless sensor networks in which the network is modeled as a hierarchical graph. In this algorithm, at each level of the hierarchical graph, a selected set of nodes is served as the message hubs (to route the messages) for the other nodes in the next level of the hierarchical graph. The proposed algorithm uses a competition-based strategy to select the nodes at each level. Dai and Wu [85] proposed a prune-based heuristic algorithm for constructing the virtual backbone of the wireless ad hoc networks by finding the dominator nodes. In this method, the connected dominating set is initialized to the set of all vertices having two unconnected neighbors. Then, the connected dominating set is modified by removing the nodes whose neighbors are also the neighbors of the other nodes in the CDS. Wu and $\mathrm{Li}$ [86] proposed a simple localized algorithm to define a connected dominating set. In this algorithm, a host is selected as a dominator if it has two unconnected neighbors. The size of the dominating set is reduced by two distributed pruning rules; a dominating node can be removed if its neighbor set is covered either by the neighbor set of an other node with higher ID or by the union of neighbor sets of two connected nodes both with higher IDs. Han [87] proposed a novel zone-based distributed algorithm for CDS formation in wireless ad hoc networks. In this Zone-based algorithm, the network is partitioned into different zones, and a dominating tree is constructed for each zone. In this method, additional nodes are inserted into the resulting connected dominating set so as to adjust the zone borders.

The virtual backbone induced by the connected dominating set forwards the packets to all the hosts within the network, while the multicast messages only should be sent to a specified group of receivers. Therefore, sending the multicast messages along the virtual backbone (CDS) imposes extra overhead too. This overhead results from a large number of unwanted rebroadcasts for sending the multicast messages to the non-member hosts. Steiner connected dominating set (SCDS) is introduced as a well-known approach to relieve such an overhead. That is, finding the Steiner connected dominating set of the network graph is a promising solution to the multicast routing problem in wireless ad hoc networks. In this method, the SCDS includes the intermediate nodes by which the massage sent out by the multicast source is relayed. The minimum Steiner connected dominating set problem was first proposed by Guha and Khuller [76], as the generalization of the well-known minimum connected dominating set problem. In this method, a (minimum size) subset of nodes is chosen as dominators to construct a route from the multicast source to each of the multicast receivers. 
The virtual backbone constructed by the Steiner connected dominating set includes the minimum number of dominator (forwarding) nodes in order to dominate the multicast members only. The following gives an overview of the methods proposed for solving the Steiner connected dominating set problem.

Wu et al. [2] proposed two approximation algorithms based on maximal independent set (MIS) for solving the minimum SCDS (MSCDS) problem. Their former algorithm is a one-hop method for approximating the MSCDS of a unit disk graph with a constant approximation ratio at most 10 . This algorithm exploits the properties of the MIS and minimum Steiner tree to form the MSCDS. The proposed algorithm first finds the MIS of the graph induced by only the vertices in multicast group. Then, the Steiner tree algorithm proposed in [88] is applied to connect the vertices of the constructed MIS. The size of the SCDS constructed by the proposed one-hop algorithm is at most $10 \times 0$ PT +1 . The time complexity of this algorithm is $O(D \cdot|V|)$, where $D$ denotes the graph diameter, and $V$ denotes the vertex set. They also proposed a $d$-hop algorithm in which a $d$-hop graph is initially constructed, where the terminals form the vertex-set of the graph. In this method, every two vertices of the graph are connected by an edge, if they are $d$-hop neighbors. Now, like the one-hop algorithm, an MIS of the $d$-hop graph is computed, and than a Steiner tree algorithm [88] is applied to connect the vertices of MIS. The $d$-hop algorithm computes a SCDS of subset $R$, whose size is at most

$$
\left(8 d^{2}+8 d+2+\frac{4}{d}\right) \cdot \mathrm{OPT}-1
$$

, if $d$ is even, and

$$
\frac{\left(16 d^{3}+16 d^{2}+4 d+6\right) \cdot \mathrm{OPT}+4 d^{3}+4 d^{2}-2 d+3}{2 d-1}
$$

if $d$ is odd. The $d$-hop algorithm can be implemented in a fully distributed manner, and the message and time complexity of the distributed $d$-hop algorithm are $O\left(|V|^{2}\right)$ and $O(D \cdot|V|)$, respectively. They showed the distributed $d$-hop algorithm can be effectively used for multicast routing in ad hoc networks by constructing a VMB with a small number of forwarding nodes. Muhammad [89, 90] also proposed a distributed MIS-based Steiner connected dominating set algorithm for multicast routing in wireless ad hoc networks. The first step of this algorithm constructs a maximal independent set in $O(|V|)$ time, whose size is at most $|\mathrm{MIS}|=4 \times \mathrm{OPT}+1$. The second step uses a distributed Steiner tree with message complexity $O(2|V|)$ and time complexity $O((|V|-1) \cdot|E| \cdot \log |V|)$ for connecting the MIS members. The total size of the SCDS constructed by Muhammad's algorithm is smaller than $2(|\mathrm{MIS}|+\mathrm{OPT}-1)+1$, and the message and time complexity of this algorithm are $O(2|V|)$ and $O((|V|-1) \cdot|E| \cdot \log |V|)$, respectively. Aggarwal et al. [4] proposed an algorithm for approximating the MSCDS in a dominating pair graph. The time complexity of the proposed algorithm is $O\left(|V|^{8} \cdot|R|\right)$, where $|V|$ and $|R|$ denotes the cardinality of the vertex set and terminal-set, respectively. The authors show that the proposed algorithm computes the Steiner connected dominating set in $O\left(|V|^{4} \cdot|R|\right)$ time, if the distance between the dominating pair vertices is greater than 8 . The cardinality of the Steiner connected dominating set constructed by Aggarwal et al.'s algorithm is smaller than OPT +2 , where $d t(G)$ is the dominating target number of graph $G$. Akbari Torkestani and Meybodi [98] presented three centralized approximation algorithms (called Algorithm I-III) for solving the minimum weight Steiner connected dominating set problem. In these algorithms, it is assumed that a random weight is associated with each node. At each stage of algorithms, a Steiner connected dominating set is randomly constructed by a network of cooperating learning automata. The weight of the selected Steiner connected dominating set is evaluated through the random environment, and depending on the response received from the environment, the selected Steiner connected dominating set is rewarded or penalized. The authors also designed a distributed version of the last proposed centralized algorithm (Algorithm III) for multicast routing in wireless mobile ad-hoc networks. The same authors [97] designed a distributed learning automata-based algorithm called MMR-LA to solve the multicast routing problem in wireless mobile Ad-hoc networks where the network topology graph 
is a stochastic graph. For the first time, they introduced the concept of stochastic Steiner connected dominating set problem and used it to form the virtual multicast backbone (VMB) of the random networks where the network parameters vary over time. The proposed algorithm uses the relative mobility of each host (with respect to all its neighbors) to predict its realistic motion behavior by sampling its movement parameters during different epochs.

\subsection{Hybrid Multicast routing protocols}

Tree-based protocols provide high data forwarding efficiency at the expense of low robustness. Their advantage is their simplicity. Their disadvantage is that until the tree is reconstructed after movement of a node, packets possibly have to be dropped. Mesh-based protocols perform better in high mobility situation as they provide redundant paths from source to destinations while forwarding data packets. However, mesh-based approaches sacrifice multicast efficiency in comparison to treebased approach. They increase the network load. Hybrid multicast routing protocols combine the both advantages of tree and mesh-based approaches, i.e., the robustness of mesh-based multicast routing protocols and low overhead of tree-based protocols. Hence, hybrid protocols address both efficiency and robustness.

In [91], Biswas et al. proposed an efficient hybrid mesh-based multicast routing protocol for ad hoc networks. The key concept is to separate data forwarding path from join-query forwarding path by incorporating low overhead local clustering technique and forwarding data packets using DDM. DDM is a stateless multicast approach where multicast tree information is appended with each data packet header. This protocol solves the scalability issues of ODMRP by reducing the control overhead and increasing multicast efficiency for different network scenarios. They incorporated a low overhead local clustering technique to classify all nodes into core and normal categories. When multicast routes to destination nodes are unavailable, join-query messages are sent to all the nodes in the network and data packets are forwarded by the core nodes to the destination nodes using differential destination multicast. Through simulations they showed that this protocol reduces the control overhead and increases packet delivery ratio by $20-50 \%$ for different network scenarios.

An and Papavassiliou [92] proposed a Mobility-based Hybrid Multicast Routing (MHMR) protocol suitable for mobile ad hoc networks. The main features of the proposed protocol are the following: (i) mobility-based clustering and group-based hierarchical structure, in order to effectively support stability and scalability; (ii) group-based (limited) mesh structure and forwarding tree concepts, in order to support the robustness of the mesh topologies, which provides limited redundancy and the efficiency of tree forwarding simultaneously; and (iii) combination of proactive and reactive concepts that provide low route acquisition delay and low overhead. Although the MHMR creates a mesh infrastructure for multicast routing, the multicast packet forwarding is done only on a source-based tree. The trees are constructed over the given mesh structure and can be created dynamically depending on the mesh stability.

Xie et al. [17] proposed an approach for robust IP multicast in mobile ad hoc networks by exploiting user-multicast tree and dynamic logical cores, called Ad hoc Multicast Routing Protocol (AMRoute). AMRoute creates a source-based multicast distribution tree using unicast tunnels connecting the group members. As a hybrid multicast routing protocol which combines advantages of tree and mesh based approaches, AMRoute is composed of a bidirectional shared multicast tree per multicast group. Certain tree nodes are designated by AMRoute as logical cores which are responsible for initiating and maintaining the signaling component of AMRoute, such as detection of group members, tree setup, and data distribution. Each group in the network has at least one logical core. In this method, unicast tunnels are used as tree links to connect neighbors on the user-multicast tree. Thus, group state cost is incurred only by group senders and receivers, and tree structure does not need to change even in case of a dynamic network topology. 


\subsection{Stateless Multicast routing protocols}

In the stateless multicast protocols, the forwarding states are included in packet header, and no protocol state is maintained at any nodes except for the multicast source node. From the information included in the packet headers, any intermediate node knows how to forward or duplicate the packet. Although packing routing information together with data traffic will enlarge data packet size, it reduces the total number of control packets generated by the protocol. Besides, when the group is idle, there is no control overhead. A recent shift toward the stateless multicasting is represented by DDM [15], LGT [93] and RDG [94]. All these protocols do not require maintenance of any routing structure at the forwarding nodes. These protocols use different techniques to achieve stateless multicasting.

In [15], a stateless multicast routing protocol termed Differential Destination Multicast (DDM) is proposed. It differs from the other approaches in two ways. First, instead of distributing membership control throughout the network, DDM concentrates this authority at the data sources (i.e., senders), thereby giving sources the knowledge of group membership. Second, differentially encoded, variablelength destination headers are inserted in data packets, which are used in combination with unicast routing tables to forward multicast packets towards multicast receivers. In DDM, a source encapsulates a list of destination addresses in the header of each data packet it sends out. When an intermediate node receives the packet, its DDM agent queries the unicast routing protocol about which next-hop node to forward the packet toward each destination in the packet header. DDM is intended for small groups, therefore, it intrinsically excels only in horizontal scalability. When group size is large, placing the addresses of all members into the packet headers will not be efficient. The protocol has a caching mode, so that only the difference from the previous states is actually placed in the headers. However, as the forwarding set at the on-route nodes inevitably grow large, each intermediate node needs to keep routes for a large set of destinations. This poses a heavy burden on the supporting unicast protocol even under moderate mobility.

Chen and Nahrstedt [93] designed a location guided tree construction Algorithm called LGT for small group multicast in mobile ad hoc networks. LGT is also a stateless multicast protocol, where multicast data is encapsulated in a unicast packet and transmitted among group members. LGT builds an overlay packet delivery tree on top of the underlying unicast routing protocol, and multicast packets are encapsulated in a unicast envelop and unicasted between the group members. In LGT, using the location information of the group member nodes, the multicast tree is constructed without the knowledge of the network topology. The authors propose two types of heuristics, namely the location-guided k-array tree (LGK), and the location-guided Steiner tree (LGS) to construct the multicast tree with location information.

Luo and Eugster [94] proposed another stateless multicast routing algorithm for wireless ad hoc networks called RDG, short for Route Driven Gossip. RDG uses a probabilistically controlled flooding technique, termed as gossiping, to deliver packets to all the group members. RDG relies on a unicast protocol such as DSR [95] to provide routing information, which is used for guiding the gossip process. Each node maintains the following data structures for each multicast group: a data buffer which stores data packets received, and a view which is a list of all other group member nodes known to this node. The view at each node is divided into two parts: active view, which contains the IDs of known members to which at least one routing path is known, and passive view which contains the IDs of known member to which no routing path is currently available. A node intending to join a group floods the network with a Group-Request message. All members receiving the message update their active view. They also return a Group-Reply to the request initiator with a certain probability. The initiator also updates its active view after receiving the Group-Reply. Each member node periodically generates a gossip message and gossips it to a set of other nodes randomly chosen from its active view. The message includes a selected subset of the data buffer, and the sequence number of the most recent missing data packets. A group member receiving a gossip message will update its view of other 
group members and update its data buffer with newly received data. In responding the gossip initiator's request of recovering the missing data, the receiving node will unicast the missing data back to the initiator if the data is in its data buffer.

\section{Conclusion}

Multicasting is used when the same message or the same stream of data must be forwarded to multiple destinations. Multicasting is an efficient data transmission method to support group-oriented communications in one-to-many or many-to-many applications such as audio/video conferencing, collaborative works, and so on. In MANETs, the most challenging issue in multicast routing is to effectively handle the frequent and unpredictable topology changes caused by host mobility, link breakage and host failure. This paper provided a survey of most recent multicast routing protocols for MANETs. This study showed that each multicast routing protocol may improve network performance in terms of delay, throughput, reliability or lifetime. Due to severe constraints of mobile wireless ad hoc networks such as host mobility, limited resources and very unreliable communication channel, single protocol or a set of protocols that can improve all these performance parameters is extremely hard to find. Selection of a multicast routing protocol is as much dependent on the nature of application, and different applications have diverse requirements. Stability against the host mobility, energy efficiency, low overhead, reliability, and scalability are several requirements for which the multicast routing protocols are designed. This survey enables the researchers to identify the strengths and weaknesses of different multicast routing protocols and helps them to choose the best one for a particular application.

\section{References}

[1] T. Nadeem, and S. Parthasarathy, "Mobility Control for Throughput Maximization in Ad hoc Networks," Wireless Communication and Mobile Computing, Vol. 6, pp. 951-967, 2006.

[2] Y. F. Wu, Y. L. Xu, C. L. Chen, and K. Wang, "On the Construction of Virtual Multicast Backbone for Wireless Ad hoc Networks," IEEE International Conference on Mobile Ad hoc and Sensor Systems, pp. 294-303, 2004.

[3] R. M. Karp, "Reducibility among Combinatorial Problems," Complexity of Computer Computations, Plenum Press, USA, pp. 85-103, 1972.

[4] D. Aggarwal, C. K. Dubey, and S. K. Mehta, "Algorithms on Graphs with Small Dominating Targets," ISAAC 2006, LNCS 4288, pp. 141-152, 2006.

[5] T. C. Chiang, C. H. Liu, and Y. M. Huang, "A Near-Optimal Multicast Scheme for Mobile Ad hoc Networks Using a Hybrid Genetic Algorithm," Expert Systems with Applications, Vol. 33 pp. 734$742,2007$.

[6] G. Rodolakis, A. Laouiti, P. Jacquet, and A. M. Naimi, "Multicast Overlay Spanning Trees in Ad hoc Networks: Capacity bounds protocol design and performance evaluation," Computer Communications, Vol. 31, pp. 1400-1412, 2008.

[7] R. Rajaraman, "Topology Control and Routing in Ad hoc Networks: A Survey," SIGACT News, Vol. 33, No. 2, pp. 60-73, 2002. 
[8] T. N. Bui, and C. M. Zrncic, "An Ant-based Algorithm for Finding Degree-Constrained Minimum Spanning Tree," in Proceedings of the 8th annual conference on Genetic and evolutionary computation, pp. 11-18, 2006.

[9] I. Chlamtac, M. Conti, J. Liu, "Mobile Ad hoc Networking: Imperatives and Challenges," Journal of Ad hoc Networks, Vol. 1, pp. 13-64, 2003.

[10] S. Basagni, M. Conti, S. Giordano and I. Stojmenovic, "Mobile Ad hoc Networking," IEEE Press, 2004.

[11] P. Mohapatra, S. Krishnamurthy, “Ad hoc Networks: Technologies and Protocols," Springer Science, 2005.

[12] T. Ozaki, J.B. Kim, and T. Suda, "Bandwidth-efficient Multicast Routing for Multi-hop Ad hoc Networks," in Proceedings of IEEE INFOCOM, Vol. 2, pp. 1182-1191, 2001.

[13] X. Zhang, L. Jacob, "MZRP: An Extension of the Zone Routing Protocol for Multicasting in MANETs," Journal of Information Science and Engineering, Vol. 20, pp. 535-551, 2004.

[14] P. Sinha, R. Sivakumar, and V. Bharghavan, "MCEDAR: Multicast Core Extraction Distributed Ad hoc Routing," IEEE Wireless Commun. and Net.Conf. (WCNC), pp. 1313-1317, 1999.

[15] L. S. Ji and M.S. Corson, "Differential Destination Multicast-a MANET Multicast Routing for Multihop Ad hoc Network, in Proceedings of IEEE INFOCOM, Vol. 2, pp. 1192-1201, 2001.

[16] C. W. Wu, Y. C. Tay, C. K. Toh, "Ad hoc Multicast Routing Protocol Utilizing Increasing Id-NumberS (AMRIS) Functional Sspecification," Internet-Draft, draft-ietf-manet-amris-spec-00.txt, 1998.

[17] J. Xie, R. Talpade, T. McAuley, and M. Liu, "AMRoute: Ad hoc Multicast Routing Protocol," ACM Mobile Networks and Applications (MONET) Journal, Vol. 7, No.6, pp. 429-439, 2002.

[18] S. J. Lee, M. Gerla, and C. C. Chiang, "On-demand Multicast Routing Protocol (ODMRP) for Ad hoc Networks," internet draft, draft-retf-manet-odmrp-01.txt, work in progress, 1999.

[19] C. Lin, and S. Chao, "A Multicast Routing Protocol for Multihop Wireless Networks," IEEE Global Telecommunication Conference (GlobeCom 1999), pp. 235-239, 1999.

[20] C. C. Chiang, M. Gerla, and L. Zhang, "Forwarding Group Multicast Protocol (FGMP) for Multihop, Mobile Wireless Networks” Journal of Cluster Computing, Vol. 1, No. 2, pp. 187-196, 1998.

[21] E. L. Madruga, J. J. Garcia, “Scalable Multicasting: The Core-Assisted Mesh Protocol," Kluwer Mobile Network, Vol. 6, No. 2, pp. 151-165, 2001.

[22] S. Basagni, I. Chlamtac, V.R. Syrotiuk, and R. Talebi, "On-demand Location Aware Multicast (OLAM) for Ad hoc Networks," in Proceedings of Wireless Communications and Networking Conference, Vol. 3, pp. 1323-1328, 2000.

[23] C. C. Hu, E. H. K. Wu, G. H. Chen, "OGHAM: On-Demand Global Hosts for Mobile Ad hoc Multicast Services,” Journal of Ad hoc Networks, Vol. 4, pp. 709-723, 2006. 
[24] E. M. Royer and C. E. Perkins, "Multicast Operation of the Ad hoc On-Demand Distance Vector Routing Protocol," in Proceedings of MobiCom, Seattle, pp. 207-218, 1999.

[25] G. D. Kondylis, S. V. Krishnamurthy, S. K. Dao, and G. J. Pottie, "Multicasting Sustained CBR and VBR Traffic in Wireless Ad hoc Networks," in Proceedings of IEEE International Conference on Communications, Vol. 1, pp. 543-549, 2000.

[26] T. Ballardie, P. Francis, and J. Crowcroft, "Core Based Trees (CBT): An Architecture for Scalable Inter-Domain Multicast Routing," in Proceedings of ACM SIGCOMM '93, pp. 85-95, 1993.

[27] D. Waitzman, C. Partridge, S. E. Deering, "Distance Vector Multicast Routing Protocol", Internet Request for Comment 1075, November 1988.

[28] A. S. Thyagarajan, and S. E. Deering, "Hierarchical Distancevector Multicast Routing for the MBone," in ACM SIGCOMM Computer Communication Review, pp.60-66, 1995.

[29] J. G. Jetcheva, and D. B. Johnson, "Adaptive Demand-driven Multicast Routing in Multi-hop Wireless Ad hoc Networks," in Proceedings of the ACM International Symposium on Mobile Ad hoc Networking and Computing (MobiHoc), 2001.

[30] C. K. Toh, G. Guichal, and S. Bunchua, “ABAM: On-Demand Associativity-Based Multicast Routing for Ad hoc Networks,” IEEE Vehicular Technology Conference, pp 987-993, 2000.

[31] E. Royer, and C. Toh, "A Review of Current Routing Protocols for Ad hoc Mobile Wireless Networks," IEEE Personal Communications, Vol. 6, No. 2, pp. 46-55, 1999.

[32] Q. Zhu, M. Parsa, and J. Garcia-Luna-Aceves, “A Source-Based Algorithm for Delay-Constrained Minimum-Cost Multicasting," IEEE INFOCOM, pp. 377-385, 1995.

[33] G. N. Rouskas, and I. Baldine, "Multicast Routing with End-to-End Delay and Delay Variation Constraints," IEEE Journal of Selected Areas in Communications, pp. 1-9, 1997.

[34] B. Wang, K. Sandeep, and S. Gupta, "Extending Lifetime of Multicast Trees in Wireless Ad hoc Networks," Journal of Information Science and Engineering, Special Issue on Mobile Computing, Vol. 20, pp. 219-243, 2004.

[35] S. Deering, D. Estrin, D. Farinacci, V. Jacobson, A. Helmy, and D. Meyer, "Protocol Independent Multicast Version 2 Dense Mode Specification," Draft-IETF-PIM-V2-DM-03.txt, 1999.

[36] J. Moy, "Multicast Routing Extensions for OSPF", Communications of the ACM, Vol. 37, No. 8, pp. 61-66, 1994.

[37] C. Perkins, and E.M. Royer, "Ad hoc on Demand Distance Vector (AODV) Routing” (internet draft), 1998.

[38] S. K. S. Gupta, P. K. Srimani, "Adaptive Core Selection and Migration Method for Multicast Routing in Mobile Ad hoc Networks," IEEE Transactions on Parallel and Distributed System, Vol. 14, No. 1, p.27-38, 2003. 
[39] B. H. Liu, P. C. Huang and M. J. Tsai, "Distributed Reformation of Core-Based Group-Shared Multicast Trees in Mobile Ad hoc Networks," Journal of Parallel and Distributed Computing, 2007, Vol. 68, pp. 582-595, 2008.

[40] Z. J. Haas, M. R. Pearlman, and P. Samar, "The Zone Routing Protocol (ZRP) for Ad hoc Networks," Draft-ietf-manet-zone-zrp-04.txt, Internet-Draft, IETF, 2002.

[41] Z. Haas, M. Pearlman, and P. Samar, "The Intrazone Routing Protocol (IARP) for Ad hoc Networks," Internet Draft: draft-ietfmanet-zone-iarp-02.txt, 2002.

[42] Z. J. Haas, M. R. Pearlman, and P. Samar, "The bordercast Resolution Protocol (BRP) for Ad hoc Networks," Draft-ietf-manet-zone-brp-02.txt, Internet-Draft, IETF, 2002.

[43] Z. J. Haas, M. R. Pearlman, and P. Samar, "The Interzone Routing Protocol (IERP) for Ad hoc Networks," Draft-ietf-manet-zone-ierp-02.txt, Internet-Draft, IETF, 2002.

[44] L. Canourgues, J. Lephay, L. Soyer, A. L. Beylot, "STAMP: Shared-Tree Ad hoc Multicast Protocol," IEEE Military Communications Conference, MILCOM 2006, pp. 1-7, 2006.

[45] B. Kaliaperumal, A. Ebenezer, Jeyakumar, “Adaptive Core Based Scalable Multicasting Networks," INDICON, 2005 Annual IEEE, pp. 198-202, 2005.

[46] D. Estrin, D. Farinacci, A. Helmy, D. Thaler, S. Deering, and M. Handley, "Protocol independent multicast-sparse mode (PIM-SM)," Protocol specification, RFC 2362, 1998.

[47] K. S. Gupta, "Cored-Based Tree with Forwarding Regions (CBT-FR): A Protocol for Reliable Multicasting in Mobile Ad hoc Networks," Journal of Parallel and Distributed Computing, Vol. 61, pp. 1249-1277, 2001.

[48] M. Dorigo, “Optimization, Learning and Natural Algorithms," Ph.D. thesis, Dipartimento di Elettronica, Politecnico di Milano, Milan, Italy, 1992.

[49] M. Dorigo, and L. M. Gambardella, “Ant Colony System: A Cooperative Learning Approach to the Traveling Salesman Problem," IEEE Transactions on Evolutionary Computation, Vol. 1, No.1, pp. 53-66, 1997.

[50] T. Stützle, and H. H. Hoos, “MAX-MIN Ant System,” Future Generation Computer Systems, Vol. 16, No.8, pp. 889-914, 2000.

[51] Y. P. Liu, M. Wu, and J. Qian, "The Distributed Multicast Routing Scheme with Delay Constraint Using Ant Colony Optimization," in Proceedings of the 6th World Congress on Intelligent Control and Automation, pp. 3062-3066, 2006.

[52] Y. Wang, and J. Xie, “Ant Colony Optimization For Multicast Routing,” IEEE APCCAS 2000,,The 2000 IEEE Asia-Pacific Conference on Circuits and Systems, pp. 54-57, 2000.

[53] Y. Zhang, H. Cai, Y. Lin, and J. Xiao, “An Ant Colony System Algorithm for the Multicast Routing Problem," Third International Conference on Natural Computation, ICNC 2007, 2007. 
[54] S. Y. Tseng, C. C. Lin, and Y.M. Huang, "Ant Colony-Based Algorithm for Constructing Broadcasting Tree with Degree and Delay Constraints," Expert Systems with Applications, Vol. 35, pp.1473$1481,2008$.

[55] Q. Zhang, Y. W. Leung, “An Orthogonal Genetic Algorithm for Multimedia Multicast Routing," IEEE Transaction on Evolutionary Computation, Vol. 3, No. 1, pp. 53-62, 1999.

[56] L. Atzori and A. Raccis, "Network Capacity Assignment for Multicast Services Using Genetic Algorithms," IEEE Communications Letters, Vol. 8, No. 6, pp. 403-405, 2004.

[57] R. H. Hwang, W. Y. Do and S. C. Yang, “Multicast Routing Based on Genetic Algorithms," Journal of Information Science and Engineering, Vol. 16, pp. 885-901, 2000.

[58] T. C. Chiang, C. H. Liu and Y.M. Huang, "A Near-Optimal Multicast Scheme for Mobile Ad hoc Networks Using a Hybrid Genetic Algorithm," Expert Systems with Applications, Vol. 33 pp. 734$742,2007$.

[59] Y. M. Huang, T. Chiang and T. W. Hou, "An Efficient Encoding Tree for Location-Aware Multicast Routing Protocol in Wireless Ad hoc Networks," Journal of Internet Technology, Vol. 6, No. 1, 2005.

[60] Y. S. Yen, Y. K. Chan, H. C. Chao, and J. H. Park, "A Genetic Algorithm for Energy-Efficient Based Multicast Routing on MANETs," Computer Communications, Vol. 31, pp. 858-869, 2008.

[61] M.S. Zahrani, M.J. Loomes, J.A. Malcolm, A.Z.M. Dayem Ullah, K. Steinhfel, and A.A. Albrecht, "Genetic Local search for Multicast Routing with Pre-Processing by Logarithmic Simulated Annealing," Computers \& Operations Research, Vol. 35, pp. 2049-2070, 2008.

[62] L. A. Zadeh, “Fuzzy sets,” Information and Control , Vol. 8, No. 3, pp. 338-353, 1965.

[63] S. J. Oh, Y. J. Kim and I. H. Bae, "A Fuzzy Multicast Method Providing Linguistic Guarantee of Disruption Free Service in Mobile Wireless Networks," Eighth International IEEE Conference on Parallel and Distributed Systems, pp. 135 - 140, 2001.

[64] T. C. Chiang and Y. M. Huang, "Multicast Routing Representation in Ad hoc Networks Using Fuzzy Petri Nets," in Proceedings of the 18th International Conference on Advanced Information Networking and Application, pp. 1-4, 2004.

[65] J. Nie, J. Wen, J. Luo, X. He, and Z. Zhou, “An Adaptive Fuzzy Logic Based Secure Routing Protocol in Mobile Ad hoc Networks," Journal of Fuzzy Sets and Systems, Vol. 157, pp. 1704-1712, 2006.

[66] B. L. Su, M. S. Wang, Y. M. Huang, "Fuzzy Logic Weighted Multi-Criteria of Dynamic Route Lifetime for Reliable Multicast Routing in ad hoc Networks," Expert Systems with Applications, Vol. 35, pp. 476-484, 2008.

[67] M. Lee, and T. Kim, "PatchODMRP: An Ad hoc Multicast Routing Protocol," in Proceedings of the 15th International Conference on Information Networking (ICOIN '01), pp. 537-543, 2001. 
[68] S. Cai, and X. Yang, "The Performance of PoolODMRP," in Proceedings of the 6th IFIP/IEEE International Conference on Management of Multimedia Networks and Service (MMNS2003), pp. 90-101, 2003.

[69] S. Cai, X. Yang, and L. Wang, "PDAODMRP: An Extended PoolODMRP Based on Passive Data Acknowledgement," Journal of Communications and Networks, Vol. 6, No. 4, pp. 362-375, 2004.

[70] S. Y. Oh, J. S. Park, and M. Gerla, "E-ODMRP: Enhanced ODMRP with Motion Adaptive Refresh," Journal of Parallel and Distributed Computing, Vol. 68, pp. 1044-1053, 2008.

[71] B. So, H. Jeon, and J. Lee, "PEODMRP: Performance Enhanced On-Demand Multicast Routing Protocol," in Proceedings of the 8th Joint Conference on Communications \& Informations (JCCI'04), Korea, 2004.

[72] Y. Zhao, L. Xu, M. Shi, “On-demand Multicast Routing Protocol with Multipoint Relay (ODMRPMPR) in Mobile Ad hoc Network," in Proceedings of International Conference on Communication Technology (ICCT 2003), pp. 1295-1300, China, 2003.

[73] J. J. Garcia-Luna-Aceves, and E. L. Madruga, “Core-Assisted Mesh Protocol,” IEEE Journal on Selected Areas in Communications, Vol. 17, No. 8, pp. 784-792, 1999.

[74] B. N. Clark, C. J. Colbourn, and D. S. Johnson, “Unit Disk Graphs,” Discrete Mathematics, Vol. 86, pp. 165-177. 1990.

[75] M. V. Marathe, H. Breu, H.B. Hunt III, S.S. Ravi, D.J. Rosenkrantz, "Simple Heuristics for Unit Disk Graphs," Networks, Vol. 25, pp. 59-68, 1995.

[76] S. Guha and S. Khuller, "Approximation Algorithms for Connected Dominating Sets," Algorithmica, Vol. 20, No. 4, pp. 374-387, 1998.

[77] S. Butenko, X. Cheng, C. Oliveira, P. M. Pardalos, "A New Heuristic for the Minimum Connected Dominating Set Problem on Ad hoc Wireless Networks," In Recent Developments in Cooperative Control and Optimization, Kluwer Academic Publishers, pp. 61-73, 2004.

[78] J. Wu, and H. Li, "On Calculating Connected Dominating Set for Efficient Routing in Ad hoc Wireless Networks," in Proceedings of the Third ACM International Workshop on Discrete Algorithms and Methods for Mobile Computing and Communications (ACM DIALM'1999), pp. 7-14, 1999.

[79] K. M. Alzoubi, P. J. Wan and O. Frieder, "Distributed Heuristics for Connected Dominating Sets in Wireless Ad hoc Networks," Journal of Communications and Networks, Vol. 4, No. 1, pp. 22-29, 2002.

[80] K. M. Alzoubi, P. J. Wan, O. Frieder, "Maximal Independent Set, Weakly Connected Dominating Set, and Induced Spanners for Mobile Ad hoc Networks," International Journal of Foundations of Computer Science, Vol. 14, No. 2, pp. 287-303, 2003. 
[81] K. M. Alzoubi, X. Y. Li, Y. Wang, P. J. Wan, and O. Frieder, "Geometric Spanners for Wireless Ad hoc Network," IEEE Transactions on Parallel and Distributed Systems, Vol. 14, No. 4, pp. 408-421, 2003.

[82] Y. Li, M. T. Thai, F. Wang, C. W. Yi, P. J. Wang, D. Z. Du, “On Greedy Construction of Connected Dominating Sets in Wireless Networks," Special issue of Wireless Communications and Mobile Computing (WCMC), 2005.

[83] B. Gao, Y. Yang, and H. Ma, "A New Distributed Approximation Algorithm for Constructing Minimum Connected Dominating Set in Wireless Ad hoc Networks," International Journal of Communication Systems, Vol. 18, pp. 743-762, 2005.

[84] R. Xie, D. Qi, Y. Li, and J. Z. Wang, "A Novel Distributed MCDS Approximation Algorithm for Wireless Sensor Networks," Journal of Wireless Communications and Mobile Computing, 2007.

[85] F. Dai and J. Wu, "An Extended Localized Algorithm for Connected Dominating Set Formation in Ad hoc Wireless Networks," IEEE Transactions on Parallel and Distributed Systems, to appear, 2004.

[86] J. Wu and H. Li, "A Dominating-Set-Based Routing Scheme in Ad hoc Wireless Networks," Telecommunication Systems Journal, Vol. 3, pp. 63-84, 2001.

[87] B. Han, "Zone-based Virtual Backbone Formation in Wireless Ad hoc Networks," Ad hoc Networks, Vol. 7, No. 1, pp.183-200, 2009.

[88] G. Singh, and K. Vellanki, “A Distributed Protocol for Constructing Multicast Trees,” in Proceedings of the International Conference on Principles of Distributed Systems, France, 1998.

[89] R. B. Muhammad, "Distributed Steiner Tree Algorithm and its Application in Ad hoc Wireless Networks," in Proceedings of The 2006 International Conference on Wireless Networks (ICWN'06), USA, pp. 173-178, 2006.

[90] R. B. Muhammad, "A Distributed Graph Algorithm for Geometric Routing in Ad hoc Wireless Networks," Journal of Networks, Vol. 2, No. 6, pp. 50-57, 2007.

[91] J. Biswas, M. Barai, S. K. Nandy, "Efficient Hybrid Multicast Routing Protocol for Ad hoc Wireless Networks," in Proceedings of 29th Annual IEEE International Conference on local computer networks, pp. 180-187, 2004.

[92] B. An, and S. Papavassiliou, "MHMR: Mobility-based Hybrid Multicast Routing Protocol in Mobile Ad hoc Wireless Networks," Wireless Communication and Mobile Computing, Vol. 3, pp. 255-270, 2003.

[93] K. Chen, and K. Nahrstedt, "Effective Location-Guided Tree Construction Algorithms for Small Group Multicast in MANET," in Proceedings of INFOCOM, pp. 1180-1189, 2002.

[94] J. Luo, P. T. Eugster, J. P. Hubaux, "Route Driven Gossip: Probabilistic Reliable Multicast in Ad hoc Networks," in Proceedings of IEEE Infocom'03, pp. 2229-2239, 2003. 
[95] J. Broch, D. B. Johnson, and D. A. Maltz, "The Dynamic Source Routing in Ad hoc Wireless Networks," Kluwer Academic Publishers, Dordrecht, pp. 153-181, 1996.

[96] J. Akbari Torkestani, and M. R. Meybodi, “A Link Stability-based Multicast Routing Protocol for Wireless Mobile Ad hoc Networks," Journal of Network and Computer Applications, Vol. 34, Issue. 4, 2011, pp. 1429-1440.

[97] J. Akbari Torkestani, and M. R. Meybodi, "Mobility-based Multicast Routing Algorithm in Wireless Mobile Ad Hoc Networks: A Learning Automata Approach," Journal of Computer Communications, Vol. 33, Issue 6, 15 April 2010, pp. 721-735.

[98] J. Akbari Torkestani, and M. R. Meybodi, "Weighted Steiner Connected Dominating Set and its Application to Multicast Routing in Wireless MANETs," Wireless Personal Communications, Springer Publishing Company, Feb. 2010. 